\title{
Article \\ Event-Triggered Asynchronous Filter of Nonlinear Switched Positive Systems with Output Quantization
}

\author{
Shitao Zhang ${ }^{1}\left(\mathbb{D}\right.$, Peng Lin ${ }^{1,2, *(\mathbb{D})}$ and Junfeng Zhang $1,2, * \mathbb{D}$ \\ 1 School of Automation, Hangzhou Dianzi University, Hangzhou 310018, China; shitaozhang@hdu.edu.cn \\ 2 Digital Economy Research Institute, Hangzhou Dianzi University, Hangzhou 310018, China \\ * Correspondence: penglin@hdu.edu.cn (P.L.); jfz@hdu.edu.cn (J.Z.)
}

Citation: Zhang, S.; Lin, P.; Zhang, J. Event-Triggered Asynchronous Filter of Nonlinear Switched Positive Systems with Output Quantization. Mathematics 2022, 10, 599. https:// doi.org/10.3390/math10040599

Academic Editor: Yueyuan Zhang

Received: 4 January 2022

Accepted: 3 February 2022

Published: 15 February 2022

Publisher's Note: MDPI stays neutral with regard to jurisdictional claims in published maps and institutional affiliations.

Copyright: (C) 2022 by the authors. Licensee MDPI, Basel, Switzerland. This article is an open access article distributed under the terms and conditions of the Creative Commons Attribution (CC BY) license (https:// creativecommons.org/licenses/by/ $4.0 /)$.

\begin{abstract}
This paper deals with a static/dynamic event-triggered asynchronous filter of nonlinear switched positive systems with output quantization. The nonlinear function is located in a sector. Both static and dynamic event-triggering conditions are established based on the 1-norm form. By virtue of the event-triggering mechanism, the error system is transformed into an interval uncertain system. An event-triggered asynchronous filter is designed by employing a matrix decomposition approach. The positivity and $L_{1}$-gain stability of the error system are guaranteed by means of linear copositive Lyapunov functions and a linear programming approach. Finally, two examples are given to verify the effectiveness of the design.
\end{abstract}

Keywords: switched nonlinear positive systems; event-triggered filter; asynchronous switching; linear programming

\section{Introduction}

As an important class of hybrid systems, switched positive systems composing of a series of positive subsystems and a switching rule to coordinate the operation of subsystems have attracted extensive attention [1,2]. Compared with the general (non-positive) switched systems [3-5], switched positive systems are more suitable to accurately model a kind of practical system consisting of nonnegative quantities, such as communication networks [6], chemical engineering [7], and water systems [8]. In [9,10], the stability and stabilization of switched positive systems were investigated based on linear copositive Lyapunov functions.

The study [11] dealt with the issue of $L_{1}$-gain characterization for switched positive systems by virtue of copositive Lyapunov function and linear programming approach. The $L_{1}$-gain analysis and control synthesis of switched positive systems was investigated in [12] using multiple linear copositive Lyapunov functions incorporated with the average dwell time approach. More results on a switched positive systems can be found in [13-15]. The above literature mainly investigated linear switched positive systems. In fact, nonlinear processes exist in most practical systems. A linear model cannot describe the nonlinear processes accurately.

Modeling such systems via nonlinear switched systems will have less error than linear switched systems. In [16], the distributed filter was proposed for nonlinear switched positive systems with stochastic nonlinearities and missing measurements based on switched Lyapunov function and linear programming. A sector nonlinearity was first introduced to ensure the positivity of nonlinear switched positive systems in $[17,18]$, where the considered nonlinear functions are located in a sector.

A robust fault detection filter was designed for nonlinear switched systems with time-varying delay based on the average dwell time approach and the Lyapunov functional technique [19]. In [20], the issue of $H_{\infty}$ filter of nonlinear switched systems with stable and unstable subsystems was solved by means of the mode-dependent average dwell time technique. Further results about nonlinear switched systems can refer to [21-25]. 
The filter design for switched systems in the literature mentioned above is mainly based on synchronous switching. It should be pointed out that it takes time to identify which subsystem is activated and which matched filter is activated. The transmission time delay of the switching signal or the impact of the external factors may result in asynchronous switching between the filters and the switched systems [26,27]. Therefore, an asynchronous filter is more practical than a synchronous one.

An asynchronous $\ell_{1}$ positive filter of switched positive systems with modal-dwelltime was proposed in [28]. Using the average dwell time and linear matrix inequality, the study [29] was concerned with the $H_{\infty}$ filtering problem of linear switched systems with asynchronous switching. An $L_{1}$-gain filter of switched positive systems was investigated by introducing a clock-dependent Lyapunov function [30]. In addition, the issue of quantization was considered in [27], which can deal with the failure phenomenon of elements. The quantization can also guarantee the safety of information transmission [31-33].

The study [31] was concerned with feedback stabilization problems for linear timeinvariant control systems with saturating quantized measurements. In [32], the authors investigated a design method of a time-varying quantizer to stabilize switched systems with quantized output and switching delays based on a dwell-time assumption and level sets of a common Lyapunov function. Using the sojourn probability-based switching law and parameterized Lyapunov functional, the literature [33] addressed the issue of quantized $H_{\infty}$ filtering for switched linear parameter-varying systems with both sojourn probabilities and unreliable communication channels. How can we establish an asynchronous filter framework of nonlinear switched positive systems and solve the signal quantization based on a linear approach? These questions motivate the current investigation.

Up to now, many related results on event-triggering mechanism have been reported in [34-38]. Event-triggered communication mechanism provides a more effective and practical method for solving the control issues than time-triggered sample to reduce unnecessary signal transmission. The study [39] investigated the event-triggered $L_{1}$-gain filter of switched positive systems subject to state saturation using linear programming and linear copositive Lyapunov function.

In [40], an event-triggered filter of positive systems was designed by adopting a matrix decomposition approach and linear copositive Lyapunov functions. An event-triggered filter of switched positive systems subject to state saturation was investigated by resorting to linear programming and average dwell time technology [41]. More recently, a dynamic event-triggered mechanism, which was developed from the static one, has been presented in $[42,43]$. In [44], the issue of recursive distributed filtering was investigated for nonlinear time-varying systems under a dynamic event-triggered mechanism.

With the help of the mathematical induction and Lyapunov theorem, the study [45] presented a dynamic event-triggered control scheme for linear time-invariant systems. The study [46] dealt with the stability of linear stochastic systems based on the dynamic eventtriggered mechanism with an impulsive switched system approach. To the best of authors' knowledge, there have been no research achievements regarding the asynchronous filter design of nonlinear switched positive systems under a static/dynamic event-triggering mechanism. Therefore, applying static and dynamic event-triggering communication mechanisms to the asynchronous filter design of switched nonlinear positive systems is one motivation of this work.

In this paper, we focus on the event-triggered $L_{1}$-gain asynchronous filter of nonlinear switched positive systems with output quantization. Static and dynamic event-triggering schemes based on 1-norm inequality are constructed for the considered systems, respectively. The filter gain matrices are designed by using the matrix decomposition technique to guarantee the positivity and $L_{1}$-gain stability of the underlying systems. The outline of the paper is as follows: Section 2 provides the problem formulation; Section 3 presents the main results; Two examples are given in Section 4; and Section 5 concludes this paper. 
Notation 1. Let $\mathbb{R}^{n}$ (or $\mathbb{R}_{+}^{n}$ ) and $\mathbb{R}^{n \times m}$ be sets of $n$-dimensional vectors (or, nonnegative) and $n \times m$ matrices, respectively. The symbols $\mathbb{N}$ and $\mathbb{N}_{+}$denote the sets of nonnegative and positive integers, respectively. For a matrix $A=\left[a_{i j}\right], A \succeq 0(\succ 0)$ indicates that $a_{i j} \geq 0\left(a_{i j}>0\right)$, $\forall i, j=1, \cdots, n$, where $a_{i j}$ is the element in the ith row and $j$ th column of $A . A^{\top}$ stands for the transpose of matrix $A$.

For $v \in \mathbb{R}^{n}, v^{(l)}$ is the $\imath$ th element of the vector. $v \succeq 0(\succ 0)$ means $v^{(\imath)} \succeq(\succ 0)$, $\forall \imath=1, \cdots, n$. The 1-norm of $x=\left(x_{1}, x_{2}, \ldots, x_{n}\right)$ is defined by $\|x\|_{1}=\sum_{l=1}^{n}\left|x_{l}\right|$, and the $\ell_{1}$ norm of the vector is $\sum_{k=0}^{\infty}\|x(k)\|_{1}$. Define $1_{n}=(\underbrace{1, \ldots, 1}_{n})^{\top} \in \mathbb{R}^{n}$ and $1_{n}^{(l)}=$ $(\underbrace{0, \ldots, 0,1}_{l}, \underbrace{0, \ldots, 0}_{n-1})^{\top}$. A matrix I denotes the identity matrix with appropriate dimensions, and $1_{n \times n} \in \mathbb{R}^{n \times n}$ is a matrix with all the elements being 1 . The logic operator $a \vee b$ means that $a$ is valid or $b$ is valid.

\section{Preliminaries}

Consider the discrete-time nonlinear switched system:

$$
\begin{gathered}
x(k+1)=A_{\sigma(k)} f(x(k))+B_{\sigma(k)} g(\omega(k)), \\
y(k)=C_{\sigma(k)} h(x(k))+D_{\sigma(k)} l(\omega(k)), \\
z(k)=E_{\sigma(k)} p(x(k))+F_{\sigma(k)} q(\omega(k)),
\end{gathered}
$$

where $x(k)=\left(x_{1}(k), \ldots, x_{n}(k)\right)^{\top} \in \mathbb{R}^{n}, y(k) \in \mathbb{R}^{m}, \omega(k) \in \mathbb{R}_{+}^{m}$, and $z(k) \in \mathbb{R}^{s}$ are the system state, measurable output, disturbance, and output to be estimated, respectively. The nonlinear functions satisfy that

$$
\begin{gathered}
f(x)=\left(f_{1}\left(x_{1}\right), \ldots, f_{n}\left(x_{n}\right)\right)^{\top}, h(x)=\left(h_{1}\left(x_{1}\right), \ldots, h_{n}\left(x_{n}\right)\right)^{\top}, \\
p(x)=\left(p_{1}\left(x_{1}\right), \ldots, p_{n}\left(x_{n}\right)\right)^{\top}, g(\omega)=\left(g_{1}\left(\omega_{1}\right), \ldots, g_{m}\left(\omega_{m}\right)\right)^{\top}, \\
l(\omega)=\left(l_{1}\left(\omega_{1}\right), \ldots, l_{m}\left(\omega_{m}\right)\right)^{\top}, q(\omega)=\left(q_{1}\left(\omega_{1}\right), \ldots, q_{m}\left(\omega_{m}\right)\right)^{\top} .
\end{gathered}
$$

The function $\sigma(k)$ denotes the switching law taking values at a finite set $S=\{1,2, \ldots, N\}$, $N \in \mathbb{N}_{+}$, where $N$ represents the number of subsystems. Assume that the $i$ th subsystem is invoked when $\sigma(k)=i$.

Assumption 1. The system matrices satisfy that $A_{i} \succeq 0, B_{i} \succeq 0, C_{i} \succeq 0, D_{i} \succeq 0, E_{i} \succeq 0$, and $F_{i} \succeq 0$ for each $i \in S$.

Assumption 2. The nonlinear functions $f(x), g(\omega), h(x), l(\omega), p(x)$, and $q(\omega)$ are located in some sector fields with

$$
\begin{aligned}
& \omega_{1} x_{i}^{2} \leq f_{i}\left(x_{i}\right) x_{i} \leq \omega_{2} x_{i}^{2}, \omega_{3} x_{i}^{2} \leq h_{i}\left(x_{i}\right) x_{i} \leq \omega_{4} x_{i}^{2}, \omega_{5} x_{i}^{2} \leq p_{i}\left(x_{i}\right) x_{i} \leq \omega_{6} x_{i}^{2}, \\
& \varepsilon_{1} \omega_{l}^{2} \leq g_{\iota}\left(\omega_{\iota}\right) \omega_{\iota} \leq \varepsilon_{2} \omega_{\iota}^{2}, \varepsilon_{3} \omega_{\iota}^{2} \leq l_{\iota}\left(\omega_{\iota}\right) \omega_{\iota} \leq \varepsilon_{4} \omega_{l}^{2}, \varepsilon_{5} \omega_{l}^{2} \leq q_{\iota}\left(\omega_{l}\right) \omega_{\iota} \leq \varepsilon_{6} \omega_{l}^{2},
\end{aligned}
$$

where $i=1,2, \cdots, n, \iota=1,2, \cdots, m, 0<\omega_{1} \leq \omega_{2}, 0<\omega_{3} \leq \omega_{4}, 0<\omega_{5} \leq \omega_{6}, 0<\varepsilon_{1} \leq$ $\varepsilon_{2}, 0<\varepsilon_{3} \leq \varepsilon_{4}, 0<\varepsilon_{5} \leq \varepsilon_{6}$, and $f_{i}(0)=0$.

Some preliminaries about positive systems are introduced.

Definition 1 ([1,2]). A system is said to be positive if all its states and outputs are nonnegative for any nonnegative initial conditions and nonnegative inputs.

Remark 1. There indeed exist some systems whose states and outputs are nonnegative for some non-positive initial conditions and inputs. The nonnegativity of these systems only holds for some of initial conditions and inputs rather than any nonnegative initial conditions and inputs. In 
this paper, the definition of positive system means that the states and outputs are nonnegative for any nonnegative initial conditions and inputs. The definition follows the notions in [1,2]. Such a definition is to guarantee the essential nonnegativity of a system for any nonnegative initial conditions and inputs. Thus, the nonnegative initial conditions are required.

Lemma $1([1,2])$. A system $x(k+1)=A x(k)$ is positive if and only if $A \succeq 0$.

Lemma 2 ([1,2]). Given a matrix $A \succeq 0$, the following conditions are equivalent:

(i) The matrix $A$ is a Schur matrix.

(ii) There exists some vector $v \succ 0$ such that $(A-I) v \prec 0$.

Definition 2 ([3]). For a switching signal $\sigma(k)$ and $0 \leq k_{1} \leq k_{2}$, denote the number of the switching of $\sigma(k)$ by $N_{\sigma}\left(k_{2}, k_{1}\right)$. If there exist $N_{0} \geq 0$ and $\tau_{a}>0$ such that

$$
N_{\sigma}\left(k_{2}, k_{1}\right) \leq N_{0}+\left(k_{2}-k_{1}\right) / \tau_{a}
$$

then $\tau_{a}$ is an average dwell time of the switching signal $\sigma(k)$.

Definition 3 ([41]). The system (1) is said to be $\ell_{1}$-gain stable if the following statements hold:

(i) For $\omega(k)=0$, the system (1) is asymptotically stable.

(ii) Under zero-initial conditions, the following inequality holds for $\omega(k) \neq 0$,

$$
\sum_{k=0}^{\infty} e^{-\hbar k}\|e(k)\|_{1} \leq \gamma \sum_{k=0}^{\infty}\|\omega(k)\|_{1},
$$

where $\gamma>0$ is the $\ell_{1}$-gain value and $\hbar>0$.

\section{Main Results}

This section first explores the positivity of system (1). Then, a nonlinear asynchronous filter is designed under static event-triggering mechanism for system (1) with output quantization. Finally, a dynamic event-triggering filter for system (1) is proposed.

\subsection{Positivity}

Lemma 3. Under Assumption 2, system (1) is positive if and only if Assumption 1 holds.

Proof. Necessity. Let $x(0)=0$. Then, $x(1)=B_{i} g(\omega(0))$ for some $i \in S$. By Assumption 2, $g(\omega(0)) \succeq 0$ for any $\omega(0) \succeq 0$. Since $x(1) \succeq 0$ for any $g(\omega(0)) \succeq 0$, then $B_{i} \succeq 0$.

Now, we prove that $A_{i} \succeq 0$ via reductio ad absurdum. Let $g(\omega(k))=0$. Suppose there exists an element $a_{i}^{(i j)}<0$, then we find

$$
x_{\imath}(k+1)=\sum_{j=1, j \neq j}^{n}\left(a_{i}^{(i j)} f_{j}\left(x_{j}(k)\right)+a_{i}^{(\imath \jmath)} f_{\jmath}\left(x_{j}(k)\right)\right) .
$$

It is possible that $x_{l}(k+1)<0$ if $a_{i}^{(\imath \jmath)}$ takes a small value enough, which yields a contradiction with the positivity of system (1). Thus, $A_{i} \succeq 0$.

Sufficiency. Denote by $\digamma$ the set of indices that satisfies $x_{\imath}(k)=0$ for $\imath \in \digamma$. Then, for some $i \in S$

$$
x_{\imath}(k+1)=\sum_{j \notin \Omega} a_{i}^{(\imath j)} f_{j}\left(x_{j}(k)\right)+\sum_{l=1}^{m} b_{i}^{(\imath l)} g_{\iota}\left(\omega_{\iota}(k)\right), \imath \in \digamma,
$$

where $a_{i}^{(\imath \jmath)}$ is the $\imath$ th row $j$ th column element of $A_{i}$ and $b_{i}^{(\imath l)}$ is the $\imath$ th row $\iota$ th column element of $B_{i}$. Note the condition (2), it follows that $f_{j}\left(x_{j}(k)\right) \geq 0$ for $k \in[0,+\infty)$. By Assumption $1, a_{i}^{(\imath \jmath)} \geq 0$ and $b_{i}^{(u)} \geq 0$. From (3), we have $g(\omega(k)) \succeq 0, l(\omega(k)) \succeq 0$, and $q(\omega(k)) \succeq 0$ for $\omega(k) \in \mathbb{R}_{+}^{m}$. So, we have $x_{\imath}(k+1) \geq 0$ for $g(\omega(k)) \succeq 0$. By (2a), we have $h(x(k)) \succeq 0$ and $p(x(k)) \succeq 0$ for $x(k) \succeq 0$. Together these with $C_{i} \succeq 0, D_{i} \succeq 0, E_{i} \succeq 0$, and $F_{i} \succeq 0$ give $y(k) \succeq 0$ and $z(k) \succeq 0$. 
The proof of Lemma 3 follows the proof of the positivity in [1,2]. The sector conditions in Assumption 2 are key to the positivity of system (1).

\subsection{Static Event-Triggering Case}

This subsection aims to design an event-triggered nonlinear asynchronous filter for a switched nonlinear positive system (1). A more general case is investigated, where the nonlinear function is unknown. This implies that the nonlinear function $f(x(k))$ cannot be used for the filter design. Thus, a nonlinear function $\hat{f}(x)$ is introduced to estimate the unknown nonlinear function $f(x)$. Then, the corresponding filter design is more difficult compared with the former filter design.

First, we introduce an event-triggering mechanism to detect and manage the transmission of output variables. Define $e_{y}(k)=\tilde{y}(k)-y(k)$, where $\tilde{y}(k)=y\left(k_{\wp}\right), y\left(k_{\wp}\right)$ is the output of event generator at the event-triggering instant $k_{\wp}, \wp \in \mathbb{N}$. The measurement output will be released only when the following event-triggering condition is satisfied:

$$
\left\|e_{y}(k)\right\|_{1}>\beta\|y(k)\|_{1}
$$

where $k \in\left[k_{\wp}, k_{\wp+1}\right)$ and $\beta \in[0,1)$ is the event-triggering coefficient.

To further reduce the design cost and increase the practicability of the filter, we introduce a quantization technique to measure the output signal. Figure 1 is the eventtriggered nonlinear quantization filter framework of switched nonlinear positive systems. The model of the quantized output signal is given as:

$$
\bar{y}(k)=\overline{\mathcal{U}}(\tilde{y}(k))=\left(\overline{\mathcal{U}}_{1}\left(\tilde{y}_{1}(k)\right), \overline{\mathcal{U}}_{2}\left(\tilde{y}_{2}(k)\right), \cdots, \overline{\mathcal{U}}_{m}\left(\tilde{y}_{m}(k)\right)\right)^{\top},
$$

where $\bar{y}(k) \in \mathbb{R}^{m}$ denotes the quantized signal of the event generator's output signal $\tilde{y}(k)$ and $\overline{\mathcal{U}}(\tilde{y}(k))$ is the logarithmic quantizer. Moreover, the subquantizer $\overline{\mathcal{U}}_{c}\left(\tilde{y}_{c}(k)\right)$ $(1 \leq c \leq m)$ is characterized by the set of quantization levels:

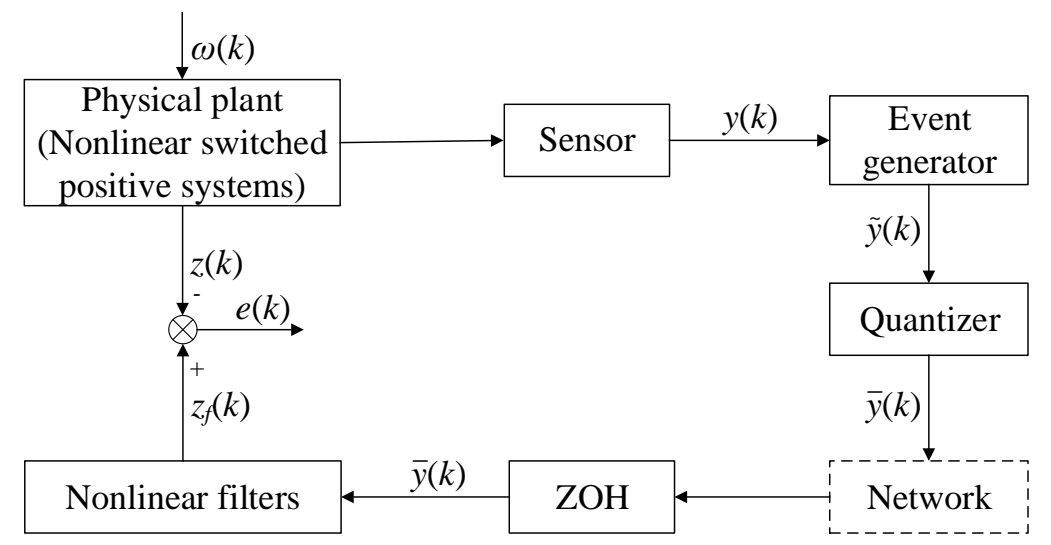

Figure 1. The event-triggered nonlinear filter with output quantization.

$$
u_{c}=\left\{\phi_{c} \mid \phi_{c}=\kappa_{c} \phi_{c 0}\right\},
$$

where $0<\kappa_{c}<1, \phi_{c 0}>0, \phi_{c}$ denotes the quantization level corresponding to a segment of $c$ th component of the output signal $\tilde{y}(k)$. Then, the subquantizer $\overline{\mathcal{U}}_{c}\left(\tilde{y}_{c}(k)\right)$ is defined as follows:

$$
\overline{\mathcal{U}}_{c}\left(\tilde{y}_{c}(k)\right)=\left\{\begin{array}{l}
\phi_{c}, \quad \frac{1}{1+\epsilon_{c}} \phi_{c}<\tilde{y}_{c}(k) \leq \frac{1}{1-\epsilon_{c}} \phi_{c} \\
0, \quad \tilde{y}_{c}(k)=0 \\
\tilde{y}_{c}(k), \quad 0<\tilde{y}_{c}(k)<\frac{1}{1+\epsilon_{c}} \phi_{c}, \tilde{y}_{c}(k)>\frac{1}{1-\epsilon_{c}} \phi_{c},
\end{array}\right.
$$


where $\epsilon_{c}=\frac{1-\kappa_{c}}{1+\kappa_{c}}$. For any quantization error, the following sector-bound expression can be obtained:

$$
\overline{\mathcal{U}}(\tilde{y}(k))-\tilde{y}(k)=\Delta(k) \tilde{y}(k),
$$

where $\Delta(k)=\operatorname{diag}\left\{\Delta_{1}(k), \Delta_{2}(k), \cdots, \Delta_{m}(k)\right\}$ and $\left|\Delta_{c}(k)\right| \leq \epsilon_{c}$. Then, the output quantization signal based on the event-triggering strategy received by the filter can be described as: $\bar{y}(k)=(I+\Delta(k)) \tilde{y}(k)$.

A nonlinear asynchronous filter with output quantization is constructed as follows:

$$
\begin{gathered}
x_{f}(k+1)=A_{f \sigma_{f}(k)} \hat{f}\left(x_{f}(k)\right)+B_{f \sigma_{f}(k)} \bar{y}(k), \\
z_{f}(k)=E_{f \sigma_{f}(k)} p\left(x_{f}(k)\right)+F_{f \sigma_{f}(k)} \bar{y}(k),
\end{gathered}
$$

where $\hat{f}\left(x_{f}(k)\right)=\left(\hat{f}_{1}\left(x_{1}\right), \ldots, \hat{f}_{n}\left(x_{n}\right)\right)^{\top}$ satisfies $\vartheta_{1} x_{i}^{2} \leq \hat{f}_{i}\left(x_{i}\right) x_{i} \leq \vartheta_{2} x_{i}^{2}$ with $\vartheta_{2}>\vartheta_{1}>0$; $x_{f}(k)$ is the state of the filter; $z_{f}(k)$ is the estimation of $z(k) ; \sigma_{f}(k)$ is switching law of the filter taking values in $S=\{1,2, \ldots, N\}$; The matrices $A_{f \sigma_{f}(k)}, B_{f \sigma_{f}(k)}, E_{f \sigma_{f}(k)}$, and $F_{f \sigma_{f}(k)}$ are to be determined.

Remark 2. Consider the interval $\left[k_{r}, k_{r+1}\right), r=0,1, \cdots$, where the asynchronous phenomenon occurs in $\left[k_{r}, k_{r}+\Delta_{r}\right)$ and the synchronous switching arises in $\left[k_{r}+\Delta_{r}, k_{r+1}\right)$. This indicates that the ith subsystem and the jth filter are active in $k \in\left[k_{r}, k_{r}+\Delta_{r}\right)$, and then the ith filter is active in $\left[k_{r}+\Delta_{r}, k_{r+1}\right)$.

Remark 3. The filter (8) is a switched system, and the matrices of the filter depend on the system modes. In this paper, the filter is assumed to be switched asynchronously with the subsystems, which means that the switching instant of the filter lags behind the system (1) by $\Delta_{r}$, where $\Delta_{0}=0$, $\Delta_{r}<k_{r+1}-k_{r}, r=1,2, \cdots$, and $k_{r}$ is the switching time instant. Therefore, $\sigma_{f}\left(k_{r}\right)=\sigma\left(k_{r}\right)+\Delta_{r}$.

Let $\widetilde{x}(k)=\left(x^{\top}(k) x_{f}^{\top}(k)-x^{\top}(k)\right)^{\top}$ and $e(k)=z_{f}(k)-z(k)$. Based on system (1) and filter (8), the following error system is obtained: For $k \in\left[k_{r}, k_{r}+\Delta_{r}\right)$,

$$
\begin{gathered}
\tilde{x}(k+1)=\left(\begin{array}{c}
A_{i} f(x(k))+B_{i} g(\omega(k)) \\
A_{f j} \hat{f}\left(x_{f}(k)\right)+B_{f j}(I+\Delta(k))\left(C_{i} h(x(k))+D_{i} l(\omega(k))+e_{y}(k)\right)-A_{i} f(x(k))-B_{i} g(\omega(k))
\end{array}\right), \\
e(k)=E_{f j} p\left(x_{f}(k)\right)+F_{f j}(I+\Delta(k))\left(C_{i} h(x(k))+D_{i} l(\omega(k))+e_{y}(k)\right)-E_{i} p(x(k))-F_{i} q(\omega(k)), \\
\text { and for } k \in\left[k_{r}+\Delta_{r}, k_{r+1}\right), \\
A_{i} f(x(k))+B_{i} g(\omega(k)) \\
\tilde{x}(k+1)=\left(\begin{array}{c}
A_{f i} \hat{f}\left(x_{f}(k)\right)+B_{f i}(I+\Delta(k))\left(C_{i} h(x(k))+D_{i} l(\omega(k))+e_{y}(k)\right)-A_{i} f(x(k))-B_{i} g(\omega(k))
\end{array}\right), \\
e(k)=E_{f i} p\left(x_{f}(k)\right)+F_{f i}(I+\Delta(k))\left(C_{i} h(x(k))+D_{i} l(\omega(k))+e_{y}(k)\right)-E_{i} p(x(k))-F_{i} q(\omega(k)) .
\end{gathered}
$$

Let $\Lambda=\operatorname{diag}\left\{\epsilon_{1}, \epsilon_{2}, \cdots, \epsilon_{m}\right\}$. Thus, we have $0 \preceq L \preceq I+\Delta(k) \preceq J$, where $L=I-\Lambda$ and $J=I+\Lambda$. Based on Assumption 2, we have that, for $k \in\left[k_{r}, k_{r}+\Delta_{r}\right)$,

$$
\begin{gathered}
\widetilde{x}(k+1) \succeq \widetilde{A}_{1 i j} \widetilde{x}(k)+\widetilde{B}_{1 i j} \omega(k)+\widetilde{D}_{1 j} e_{y}(k), \\
e(k) \succeq \widetilde{E}_{1 i j} \widetilde{x}(k)+\widetilde{F}_{1 i j} \omega(k)+F_{f j} L e_{y}(k),
\end{gathered}
$$

and

$$
\begin{gathered}
\widetilde{x}(k+1) \preceq \widetilde{A}_{2 i j} \widetilde{x}(k)+\widetilde{B}_{2 i j} \omega(k)+\widetilde{D}_{2 j} e_{y}(k), \\
e(k) \preceq \widetilde{E}_{2 i j} \widetilde{x}(k)+\widetilde{F}_{2 i j} \omega(k)+F_{f j} J e_{y}(k),
\end{gathered}
$$


where

$$
\begin{aligned}
& \widetilde{A}_{1 i j}=\left(\begin{array}{cc}
\omega_{1} A_{i} & 0 \\
\vartheta_{1} A_{f j}+\omega_{3} B_{f j} L C_{i}-\omega_{2} A_{i} & \vartheta_{1} A_{f j}
\end{array}\right), \\
& \widetilde{A}_{2 i j}=\left(\begin{array}{cc}
\omega_{2} A_{i} & 0 \\
\vartheta_{2} A_{f j}+\omega_{4} B_{f j} J C_{i}-\omega_{1} A_{i} & \vartheta_{2} A_{f j}
\end{array}\right) \text {, } \\
& \widetilde{E}_{1 i j}=\left(\omega_{5} E_{f j}+\omega_{3} F_{f j} L C_{i}-\omega_{6} E_{i} \quad \omega_{5} E_{f j}\right), \\
& \widetilde{E}_{2 i j}=\left(\begin{array}{lll}
\omega_{6} E_{f j}+\omega_{4} F_{f j} J C_{i}-\omega_{5} E_{i} & \omega_{6} E_{f j}
\end{array}\right), \\
& \widetilde{B}_{1 i j}=\left(\begin{array}{c}
\varepsilon_{1} B_{i} \\
\varepsilon_{3} B_{f j} L D_{i}-\varepsilon_{2} B_{i}
\end{array}\right), \widetilde{B}_{2 i j}=\left(\begin{array}{c}
\varepsilon_{2} B_{i} \\
\varepsilon_{4} B_{f j} J D_{i}-\varepsilon_{1} B_{i}
\end{array}\right) \text {, } \\
& \widetilde{F}_{1 i j}=\left(\varepsilon_{3} F_{f j} L D_{i}-\varepsilon_{6} F_{i}\right), \widetilde{F}_{2 i j}=\left(\varepsilon_{4} F_{f j} J D_{i}-\varepsilon_{5} F_{i}\right), \\
& \widetilde{D}_{1 j}=\left(\begin{array}{c}
0 \\
B_{f j} L
\end{array}\right), \widetilde{D}_{2 j}=\left(\begin{array}{c}
0 \\
B_{f j} J
\end{array}\right) \text {, }
\end{aligned}
$$

and for $k \in\left[k_{r}+\Delta_{r}, k_{r+1}\right)$,

$$
\begin{gathered}
\widetilde{x}(k+1) \succeq \widetilde{A}_{1 i} \widetilde{x}(k)+\widetilde{B}_{1 i} \omega(k)+\widetilde{D}_{1 i} e_{y}(k), \\
e(k) \succeq \widetilde{E}_{1 i} \widetilde{x}(k)+\widetilde{F}_{1 i} \omega(k)+F_{f i} L e_{y}(k),
\end{gathered}
$$

and

$$
\begin{gathered}
\widetilde{x}(k+1) \preceq \widetilde{A}_{2 i} \widetilde{x}(k)+\widetilde{B}_{2 i} \omega(k)+\widetilde{D}_{2 i} e_{y}(k), \\
e(k) \preceq \widetilde{E}_{2 i} \widetilde{x}(k)+\widetilde{F}_{2 i} \omega(k)+F_{f i} J e_{y}(k),
\end{gathered}
$$

where

$$
\begin{aligned}
& \widetilde{A}_{1 i}=\left(\begin{array}{cc}
\omega_{1} A_{i} & 0 \\
\vartheta_{1} A_{f i}+\omega_{3} B_{f i} L C_{i}-\omega_{2} A_{i} & \vartheta_{1} A_{f i}
\end{array}\right), \\
& \widetilde{A}_{2 i}=\left(\begin{array}{cc}
\omega_{2} A_{i} & 0 \\
\vartheta_{2} A_{f i}+\omega_{4} B_{f i} J C_{i}-\omega_{1} A_{i} & \vartheta_{2} A_{f i}
\end{array}\right), \\
& \widetilde{E}_{1 i}=\left(\begin{array}{cc}
\omega_{5} E_{f i}+\omega_{3} F_{f i} L C_{i}-\omega_{6} E_{i} & \omega_{5} E_{f i}
\end{array}\right), \\
& \widetilde{E}_{2 i}=\left(\omega_{6} E_{f i}+\omega_{4} F_{f i} J C_{i}-\omega_{5} E_{i} \omega_{6} E_{f i}\right), \\
& \widetilde{B}_{1 i}=\left(\begin{array}{c}
\varepsilon_{1} B_{i} \\
\varepsilon_{3} B_{f i} L D_{i}-\varepsilon_{2} B_{i}
\end{array}\right), \widetilde{B}_{2 i}=\left(\begin{array}{c}
\varepsilon_{2} B_{i} \\
\varepsilon_{4} B_{f i} J D_{i}-\varepsilon_{1} B_{i}
\end{array}\right), \\
& \widetilde{F}_{1 i}=\left(\varepsilon_{3} F_{f i} L D_{i}-\varepsilon_{6} F_{i}\right), \widetilde{F}_{2 i}=\left(\varepsilon_{4} F_{f i} J D_{i}-\varepsilon_{5} F_{i}\right), \\
& \widetilde{D}_{1 i}=\left(\begin{array}{c}
0 \\
B_{f i} L
\end{array}\right), \widetilde{D}_{2 i}=\left(\begin{array}{c}
0 \\
B_{f i} J
\end{array}\right) \text {. }
\end{aligned}
$$

Theorem 1. If there exist constants $0<\omega_{1} \leq \omega_{2}, 0<\omega_{3} \leq \omega_{4}, 0<\omega_{5} \leq \omega_{6}, 0<\varepsilon_{1} \leq \varepsilon_{2}$, $0<\varepsilon_{3} \leq \varepsilon_{4}, 0<\varepsilon_{5} \leq \varepsilon_{6}, 0<\vartheta_{1} \leq \vartheta_{2}, \gamma>0, \lambda>1,0 \leq \beta<1,0<\mu_{1}<1, \mu_{2}>1, \mathbb{R}^{n}$ vectors $\zeta_{i} \succ 0, \zeta_{(i, j)} \succ 0, \varphi_{i} \succ 0, \varphi_{(i, j)} \succ 0, \xi_{i} \succeq 0, \xi_{i l} \succeq 0, \xi_{j} \succeq 0, \xi_{j l} \succeq 0, \rho_{i j} \succeq 0$, and $\mathbb{R}^{m}$ vectors $\delta_{i} \succeq 0, \delta_{i \imath} \succeq 0, \delta_{j} \succeq 0, \delta_{j l} \succeq 0, \theta_{i j} \succeq 0$ such that

$$
\begin{gathered}
\vartheta_{1} \sum_{l=1}^{n} 1_{n}^{(\imath)} \xi_{i l}^{\top}+\omega_{3} \sum_{l=1}^{n} 1_{n}^{(\imath)} \delta_{i l}^{\top} L M C_{i}-\omega_{2} 1_{n}^{\top} \varphi_{i} A_{i} \succeq 0, \\
\varepsilon_{3} \sum_{l=1}^{n} 1_{n}^{(l)} \delta_{i l}^{\top} L M D_{i}-\varepsilon_{2} 1_{n}^{\top} \varphi_{i} B_{i} \succeq 0, \\
\omega_{5} \sum_{j=1}^{s} 1_{s}^{(\jmath)} \rho_{i \jmath}^{\top}+\omega_{3} \sum_{j=1}^{s} 1_{s}^{(\jmath)} \theta_{i j}^{\top} L M C_{i}-\omega_{6} E_{i} \succeq 0, \\
\varepsilon_{3} \sum_{j=1}^{s} 1_{s}^{(\jmath)} \theta_{i j}^{\top} L M D_{i}-\varepsilon_{6} F_{i} \succeq 0, \\
\vartheta_{1} \sum_{l=1}^{n} 1_{n}^{(l)} \xi_{j l}^{\top}+\omega_{3} \sum_{l=1}^{n} 1_{n}^{(l)} \delta_{j l}^{\top} L M C_{i}-\omega_{2} 1_{n}^{\top} \varphi_{j} A_{i} \succeq 0, \\
\varepsilon_{3} \sum_{l=1}^{n} 1_{n}^{(l)} \delta_{j l}^{\top} L M D_{i}-\varepsilon_{2} 1_{n}^{\top} \varphi_{j} B_{i} \succeq 0, \\
\omega_{5} \sum_{j=1}^{s} 1_{s}^{(j)} \rho_{j \jmath}^{\top}+\omega_{3} \sum_{j=1}^{s} 1_{s}^{(\jmath)} \theta_{j j}^{\top} L M C_{i}-\omega_{6} E_{i} \succeq 0, \\
\varepsilon_{3} \sum_{j=1}^{s} 1_{s}^{(\jmath)} \theta_{j j}^{\top} L M D_{i}-\varepsilon_{6} F_{i} \succeq 0,
\end{gathered}
$$




$$
\begin{aligned}
& \omega_{2} A_{i}^{\top} \zeta_{i}+\vartheta_{2} \xi_{i}+\omega_{4} C_{i}^{\top} H J \delta_{i}-\omega_{1} A_{i}^{\top} \varphi_{i}-\mu_{1} \zeta_{i}+\left(\omega_{6} \sum_{\jmath=1}^{s} 1_{s}^{(j)} \rho_{i j}^{\top}\right. \\
& \left.+\omega_{4} \sum_{\jmath=1}^{s} 1_{s}^{(\jmath)} \theta_{i j}^{\top} J H C_{i}-\omega_{5} E_{i}\right)^{\top} 1_{s} \preceq 0, \\
& \omega_{6}\left(\sum_{\jmath=1}^{s} 1_{s}^{(\jmath)} \rho_{i \jmath}^{\top}\right)^{\top} 1_{s}+\vartheta_{2} \xi_{i}-\mu_{1} \varphi_{i} \preceq 0, \\
& \varepsilon_{2} B_{i}^{\top} \zeta_{i}+\varepsilon_{4} D_{i}^{\top} H J \delta_{i}-\varepsilon_{1} B_{i}^{\top} \varphi_{i}+\varepsilon_{4} D_{i}^{\top} H J\left(\sum_{\jmath=1}^{s} 1_{s}^{(j)} \theta_{i j}^{\top}\right)^{\top} 1_{s}-\varepsilon_{5} F_{i}^{\top} 1_{s}-\gamma 1_{m} \preceq 0 \text {, } \\
& \omega_{2} A_{i}^{\top} \zeta_{(i, j)}+\vartheta_{2} \xi_{j}+\omega_{4} C_{i}^{\top} H J \delta_{j}-\omega_{1} A_{i}^{\top} \varphi_{(i, j)}-\mu_{2} \zeta_{(i, j)}+\left(\omega_{6} \sum_{j=1}^{s} 1_{s}^{(j)} \rho_{j \jmath}^{\top}\right. \\
& \left.+\omega_{4} \sum_{\jmath=1}^{s} 1_{s}^{(\jmath)} \theta_{j \jmath}^{\top} J H C_{i}-\omega_{5} E_{i}\right)^{\top} 1_{s} \preceq 0, \\
& \omega_{6}\left(\sum_{\jmath=1}^{s} 1_{s}^{(j)} \rho_{j j}^{\top}\right)^{\top} 1_{s}+\vartheta_{2} \xi_{j}-\mu_{2} \varphi_{(i, j)} \preceq 0, \\
& \varepsilon_{2} B_{i}^{\top} \zeta_{(i, j)}+\varepsilon_{4} D_{i}^{\top} H J \delta_{j}-\varepsilon_{1} B_{i}^{\top} \varphi_{(i, j)}+\varepsilon_{4} D_{i}^{\top} H J\left(\sum_{j=1}^{s} 1_{s}^{(j)} \theta_{j \jmath}^{\top}\right)^{\top} 1_{s} \\
& -\varepsilon_{5} F_{i}^{\top} 1_{s}-\gamma 1_{m} \preceq 0, \\
& \zeta_{i} \preceq \lambda \zeta_{(i, j)}, \zeta_{i} \preceq \lambda \zeta_{(j, i)}, \zeta_{(i, j)} \preceq \lambda \zeta_{i}, \zeta_{(j, i)} \preceq \lambda \zeta_{i}, \\
& \varphi_{i} \preceq \lambda \varphi_{(i, j)}, \varphi_{i} \preceq \lambda \varphi_{(j, i)}, \varphi_{(i, j)} \preceq \lambda \varphi_{i}, \varphi_{(j, i)} \preceq \lambda \varphi_{i}, \\
& \xi_{i l} \preceq \xi_{i}, \delta_{i l} \preceq \delta_{i}, \xi_{j l} \preceq \xi_{j}, \delta_{j l} \preceq \delta_{j},
\end{aligned}
$$

hold $\forall i, j \in S, i \neq j, \imath=1,2, \cdots, n$ and $\jmath=1,2, \cdots, s$, then the error systems (9) and (10) are positive and stable with filter gain matrices

$$
\begin{gathered}
A_{f i}=\frac{\sum_{l=1}^{n} 1_{n}^{(l)} \xi_{i l}^{\top}}{1_{n}^{\top} \varphi_{i}}, B_{f i}=\frac{\sum_{l=1}^{n} 1_{n}^{(l)} \delta_{i l}^{\top}}{1_{n}^{\top} \varphi_{i}}, \\
E_{f i}=\sum_{j=1}^{s} 1_{s}^{(j)} \rho_{i j}^{\top}, F_{f i}=\sum_{j=1}^{s} 1_{s}^{(j)} \theta_{i j}^{\top},
\end{gathered}
$$

and the switching law satisfying

$$
\begin{aligned}
& \frac{\Gamma^{-}\left(k_{0}, k\right)}{\Gamma^{+}\left(k_{0}, k\right)} \geq \frac{\ln \mu_{2}-\ln \mu_{1}}{\ln \mu_{1}^{*}-\ln \mu_{1}}, \mu_{1}^{*} \in\left(\mu_{1}, 1\right), \\
& \tau_{a} \geq \tau_{a}^{*}=-\frac{2 \ln \lambda+\left(\ln \mu_{2}-\ln \mu_{1}\right) \Delta_{\max }}{\ln \mu_{1}^{*}},
\end{aligned}
$$

where $M=I-\beta 1_{m \times m}, H=I+\beta 1_{m \times m}$, and $\Delta_{\max }$ denotes the maximum of time lag $\Delta_{r}$.

Proof. First, the positivity of the error systems (9) and (10) are considered. For $x\left(k_{0}\right) \succeq 0$, the output satisfies $y\left(k_{0}\right) \succeq 0$. Using event-triggering condition (6) gives

$$
\left\|e_{y}\left(k_{0}\right)\right\|_{1} \leq \beta 1_{m}^{\top} y\left(k_{0}\right)
$$

which gives that

$$
-\beta 1_{m \times m} y\left(k_{0}\right) \preceq e_{y}\left(k_{0}\right) \preceq \beta 1_{m \times m} y\left(k_{0}\right) .
$$

For $k \in\left[k_{r}, k_{r}+\Delta_{r}\right)$, it follows from (11) and (36) that

$$
\begin{aligned}
& \widetilde{x}\left(k_{0}+1\right) \succeq \widetilde{\widetilde{A}}_{1 i j} \widetilde{x}\left(k_{0}\right)+\underline{\widetilde{B}}_{1 i j} \omega\left(k_{0}\right), \\
& e\left(k_{0}\right) \succeq \underline{\widetilde{E}}_{1 i j} \widetilde{x}\left(k_{0}\right)+\underline{\widetilde{F}}_{1 i j} \omega\left(k_{0}\right),
\end{aligned}
$$

where

$$
\begin{gathered}
\underline{\widetilde{A}}_{1 i j}=\left(\begin{array}{cc}
\omega_{1} A_{i} & 0 \\
\vartheta_{1} A_{f j}+\omega_{3} B_{f j} L M C_{i}-\omega_{2} A_{i} & \vartheta_{1} A_{f j}
\end{array}\right), \underline{\widetilde{B}}_{1 i j}=\left(\begin{array}{c}
\varepsilon_{1} B_{i} \\
\varepsilon_{3} B_{f j} L M D_{i}-\varepsilon_{2} B_{i}
\end{array}\right), \\
\underline{\widetilde{E}}_{1 i j}=\left(\begin{array}{ll}
\omega_{5} E_{f j}+\omega_{3} F_{f j} L M C_{i}-\omega_{6} E_{i} & \omega_{5} E_{f j}
\end{array}\right), \widetilde{\underline{F}}_{1 i j}=\left(\varepsilon_{3} F_{f j} L M D_{i}-\varepsilon_{6} F_{i}\right) .
\end{gathered}
$$


For $k \in\left[k_{r}+\Delta_{r}, k_{r+1}\right)$,

$$
\begin{aligned}
& \widetilde{x}\left(k_{0}+1\right) \succeq \widetilde{A}_{1 i} \widetilde{x}\left(k_{0}\right)+\widetilde{B}_{1 i} \omega\left(k_{0}\right), \\
& e\left(k_{0}\right) \succeq \underline{E}_{1 i} \widetilde{x}\left(k_{0}\right)+\underline{\widetilde{E}}_{1 i} \omega\left(k_{0}\right),
\end{aligned}
$$

where

$$
\begin{aligned}
& \underline{\widetilde{A}}_{1 i}=\left(\begin{array}{cc}
\omega_{1} A_{i} & 0 \\
\vartheta_{1} A_{f i}+\omega_{3} B_{f i} L M C_{i}-\omega_{2} A_{i} & \vartheta_{1} A_{f i}
\end{array}\right), \underline{\widetilde{B}}_{1 i}=\left(\begin{array}{c}
\varepsilon_{1} B_{i} \\
\varepsilon_{3} B_{f i} L M D_{i}-\varepsilon_{2} B_{i}
\end{array}\right), \\
& \widetilde{\widetilde{E}}_{1 i}=\left(\omega_{5} E_{f i}+\omega_{3} F_{f i} L M C_{i}-\omega_{6} E_{i} \quad \omega_{5} E_{f i}\right), \underline{\widetilde{F}}_{1 i}=\left(\varepsilon_{3} F_{f i} L M D_{i}-\varepsilon_{6} F_{i}\right) \text {. }
\end{aligned}
$$

Using (15), (16), (19) and (20) gives

$$
\begin{gathered}
\frac{\vartheta_{1} \sum_{l=1}^{n} 1_{n}^{(l)} \xi_{i t}^{\top}}{1_{n}^{\top} \varphi_{i}}+\frac{\omega_{3} \sum_{l=1}^{n} 1_{n}^{(l)} \delta_{i i}^{\top}}{1_{n}^{\top} \varphi_{i}} L M C_{i}-\omega_{2} A_{i} \succeq 0, \\
\frac{\varepsilon_{3} \sum_{l=1}^{n} 1_{n}^{(l)} \delta_{i i}^{\top}}{1_{n}^{\top} \varphi_{i}} L M D_{i}-\varepsilon_{2} B_{i} \succeq 0, \\
\frac{\vartheta_{1} \sum_{l=1}^{n} 1_{n}^{(l)} \xi_{j l}^{\top}}{1_{n}^{\top} \varphi_{j}}+\frac{\omega_{3} \sum_{l=1}^{n} 1_{n}^{(1)} \delta_{j t}^{\top}}{1_{n}^{\top} \varphi_{j}} L M C_{i}-\omega_{2} A_{i} \succeq 0, \\
\frac{\varepsilon_{3} \sum_{l=1}^{n} 1_{n}^{(l)} \delta_{j t}^{\top}}{1_{n}^{\top} \varphi_{j}} L M D_{i}-\varepsilon_{2} B_{i} \succeq 0 .
\end{gathered}
$$

Together with (17), (18), (21), (22), (31), and (32), we have

$$
\begin{gathered}
\vartheta_{1} A_{f i}+\omega_{3} B_{f i} L M C_{i}-\omega_{2} A_{i} \succeq 0, \\
\varepsilon_{3} B_{f i} L M D_{i}-\varepsilon_{2} B_{i} \succeq 0, \\
\omega_{5} E_{f i}+\omega_{3} F_{f i} L M C_{i}-\omega_{6} E_{i} \succeq 0, \\
\varepsilon_{3} F_{f i} L M D_{i}-\varepsilon_{6} F_{i} \succeq 0, \\
\vartheta_{1} A_{f j}+\omega_{3} B_{f j} L M C_{i}-\omega_{2} A_{i} \succeq 0, \\
\varepsilon_{3} B_{f j} L M D_{i}-\varepsilon_{2} B_{i} \succeq 0, \\
\omega_{5} E_{f j}+\omega_{3} F_{f j} L M C_{i}-\omega_{6} E_{i} \succeq 0, \\
\varepsilon_{3} F_{f j} L M D_{i}-\varepsilon_{6} F_{i} \succeq 0 .
\end{gathered}
$$

Due to $\xi_{i l} \succeq 0, \delta_{i l} \succeq 0, \rho_{i j} \succeq 0$, and $\theta_{i j} \succeq 0$, this yields $A_{f i} \succeq 0, B_{f i} \succeq 0, E_{f i} \succeq 0$, and $F_{f i} \succeq 0$. Thus, we have $\underline{\widetilde{A}}_{1 i} \succeq 0, \underline{\widetilde{B}}_{1 i} \succeq 0, \underline{\widetilde{E}}_{1 i} \succeq 0$, and $\underline{\widetilde{E}}_{1 i} \succeq 0$. Similarly, we can obtain $\underline{\widetilde{A}}_{1 i j} \succeq 0, \underline{\widetilde{B}}_{1 i j} \succeq 0, \widetilde{E}_{1 i j} \succeq 0$, and $\underline{\widetilde{F}}_{1 i j} \succeq 0$. By (37), (38), and Lemma 1, we have $\widetilde{x}\left(k_{0}+1\right) \succeq 0$ and $e\left(k_{0}\right) \succeq 0$. Using recursive derivation gives $\widetilde{x}(k) \succeq 0$ and $e(k) \succeq 0$, that is to say, the error systems (9) and (10) are positive.

Next, we will analyze the $\ell_{1}$-gain stability of the considered error systems. Construct a piecewise multiple copositive Lyapunov function candidate:

$$
V_{i}(k)=\left\{\begin{array}{l}
\widetilde{x}^{\top}(k) v_{i}, \forall k \in\left[k_{r-1}+\Delta_{r-1}, k_{r}\right), \\
\widetilde{x}^{\top}(k) v_{(i, j)}, \forall k \in\left[k_{r}, k_{r}+\Delta_{r}\right),
\end{array}\right.
$$

where $v_{i}=\left(\zeta_{i}^{\top} \varphi_{i}^{\top}\right)^{\top}$ and $v_{(i, j)}=\left(\zeta_{(i, j)}^{\top} \varphi_{(i, j)}^{\top}\right)^{\top}$. From (12) and (36), for $k \in\left[k_{r}, k_{r}+\Delta_{r}\right)$, it follows that

$$
\begin{gathered}
\widetilde{x}(k+1) \preceq \overline{\widetilde{A}}_{1 i j} \widetilde{x}(k)+\overline{\widetilde{B}}_{1 i j} \omega(k), \\
e(k) \preceq \overline{\widetilde{E}}_{1 i j} \widetilde{x}(k)+\overline{\widetilde{F}}_{1 i j} \omega(k),
\end{gathered}
$$


where

$$
\begin{aligned}
& \overline{\widetilde{A}}_{1 i j}=\left(\begin{array}{cc}
\omega_{2} A_{i} & 0 \\
\vartheta_{2} A_{f j}+\omega_{4} B_{f j} J H C_{i}-\omega_{1} A_{i} & \vartheta_{2} A_{f j}
\end{array}\right), \overline{\widetilde{B}}_{1 i j}=\left(\begin{array}{c}
\varepsilon_{2} B_{i} \\
\varepsilon_{4} B_{f j} J H D_{i}-\varepsilon_{1} B_{i}
\end{array}\right), \\
& \widetilde{\widetilde{E}}_{1 i j}=\left(\omega_{6} E_{f j}+\omega_{4} F_{f j} J H C_{i}-\omega_{5} E_{i} \quad \omega_{6} E_{f j}\right), \widetilde{\widetilde{F}}_{1 i j}=\left(\varepsilon_{4} F_{f j} J H D_{i}-\varepsilon_{5} F_{i}\right) \text {. }
\end{aligned}
$$

For $k \in\left[k_{r}+\Delta_{r}, k_{r+1}\right)$, we have

$$
\begin{gathered}
\tilde{x}(k+1) \preceq \overline{\widetilde{A}}_{1 i} \widetilde{x}(k)+\overline{\widetilde{B}}_{1 i} \omega(k), \\
e(k) \preceq \widetilde{\widetilde{E}}_{1 i} \widetilde{x}(k)+\overline{\widetilde{F}}_{1 i} \omega(k),
\end{gathered}
$$

where

$$
\begin{aligned}
& \overline{\widetilde{A}}_{1 i}=\left(\begin{array}{cc}
\omega_{2} A_{i} & 0 \\
\vartheta_{2} A_{f i}+\omega_{4} B_{f i} J H C_{i}-\omega_{1} A_{i} & \vartheta_{2} A_{f i}
\end{array}\right), \overline{\widetilde{B}}_{1 i}=\left(\begin{array}{c}
\varepsilon_{2} B_{i} \\
\varepsilon_{4} B_{f i} J H D_{i}-\varepsilon_{1} B_{i}
\end{array}\right), \\
& \widetilde{\widetilde{E}}_{1 i}=\left(\omega_{6} E_{f i}+\omega_{4} F_{f i} J H C_{i}-\omega_{5} E_{i} \quad \omega_{6} E_{f i}\right), \widetilde{\widetilde{F}}_{1 i}=\left(\varepsilon_{4} F_{f i} J H D_{i}-\varepsilon_{5} F_{i}\right) \text {. }
\end{aligned}
$$

From the upper bound systems (52) and (53), the forward difference of (51) along the trajectories satisfies

$$
\Delta V_{i}(k) \leq\left\{\begin{array}{l}
\tilde{x}^{\top}(k) \mathrm{Y}_{1}+\omega^{\top}(k) \Theta_{1}, \quad \forall k \in\left[k_{r-1}+\Delta_{r-1}, k_{r}\right), \\
\tilde{x}^{\top}(k) \mathrm{Y}_{2}+\omega^{\top}(k) \Theta_{2}, \forall k \in\left[k_{r}, k_{r}+\Delta_{r}\right),
\end{array}\right.
$$

where

$$
\begin{gathered}
\mathrm{Y}_{1}=\overline{\widetilde{A}}_{1 i}^{\top} v_{i}-v_{i}=\left(\begin{array}{c}
\omega_{2} A_{i}^{\top} \zeta_{i}+\left(\vartheta_{2} A_{f i}^{\top}+\omega_{4} C_{i}^{\top} H J B_{f i}^{\top}-\omega_{1} A_{i}^{\top}\right) \varphi_{i}-\zeta_{i} \\
\vartheta_{2} A_{f i}^{\top} \varphi_{i}-\varphi_{i}
\end{array}\right), \\
\mathrm{Y}_{2}=\overline{\widetilde{A}}_{1 i j}^{\top} v_{(i, j)}-v_{(i, j)}=\left(\begin{array}{c}
\left.\omega_{2} A_{i}^{\top} \zeta_{(i, j)}+\left(\vartheta_{2} A_{f j}^{\top}+\omega_{4} C_{i}^{\top} H J B_{f j}^{\top}-\omega_{1} A_{i}^{\top}\right) \varphi_{(i, j)}-\zeta_{(i, j)}\right), \\
\vartheta_{2} A_{f j}^{\top} \varphi_{(i, j)}-\varphi_{(i, j)}
\end{array}\right), \\
\Theta_{1}=\widetilde{\widetilde{B}}_{1 i}^{\top} v_{i}=\left(\varepsilon_{2} B_{i}^{\top} \zeta_{i}+\left(\varepsilon_{4} D_{i}^{\top} H J B_{f i}^{\top}-\varepsilon_{1} B_{i}^{\top}\right) \varphi_{i}\right), \\
\Theta_{2}=\overline{\widetilde{B}}_{1 i j}^{\top} v_{(i, j)}=\left(\varepsilon_{2} B_{i}^{\top} \zeta_{(i, j)}+\left(\varepsilon_{4} D_{i}^{\top} H J B_{f j}^{\top}-\varepsilon_{1} B_{i}^{\top}\right) \varphi_{(i, j)}\right) .
\end{gathered}
$$

Using (30) and (31), it derives that

$$
A_{f i} \preceq \frac{\sum_{l=1}^{n} 1_{n}^{(l)} \xi_{i}^{\top}}{1_{n}^{\top} \varphi_{i}}=\frac{1_{n} \xi_{i}^{\top}}{1_{n}^{\top} \varphi_{i}}, B_{f i} \preceq \frac{\sum_{l=1}^{n} 1_{n}^{(l)} \delta_{i}^{\top}}{1_{n}^{\top} \varphi_{i}}=\frac{1_{n} \delta_{i}^{\top}}{1_{n}^{\top} \varphi_{i}} .
$$

Together with the fact $\varphi_{i} \succ 0$ gives

$$
A_{f i}^{\top} \varphi_{i} \preceq \frac{\xi_{i} 1_{n}^{\top}}{1_{n}^{\top} \varphi_{i}} \varphi_{i}=\xi_{i}, B_{f i}^{\top} \varphi_{i} \preceq \frac{\delta_{i} 1_{n}^{\top}}{1_{n}^{\top} \varphi_{i}} \varphi_{i}=\delta_{i} .
$$

Similarly,

$$
A_{f j}^{\top} \varphi_{(i, j)} \preceq \frac{\xi_{j} 1_{n}^{\top}}{1_{n}^{\top} \varphi_{(i, j)}} \varphi_{(i, j)}=\xi_{j}, B_{f j}^{\top} \varphi_{(i, j)} \preceq \frac{\delta_{j} 1_{n}^{\top}}{1_{n}^{\top} \varphi_{(i, j)}} \varphi_{(i, j)}=\delta_{j} .
$$

Define $\Xi(k)=\gamma\|\omega(k)\|_{1}-\|e(k)\|_{1}$. From (23)-(28), (54), (55), and (56), we can obtain

$$
V_{i}(k) \leq\left\{\begin{array}{l}
\mu_{1} V_{i}(k-1)+\Xi(k-1), \forall k \in\left[k_{r-1}+\Delta_{r-1}, k_{r}\right), \\
\mu_{2} V_{(i, j)}(k-1)+\Xi(k-1), \forall k \in\left[k_{r}, k_{r}+\Delta_{r}\right) .
\end{array}\right.
$$

Thus, 


$$
\begin{aligned}
& V_{i}(k) \leq\left\{\begin{array}{l}
\mu_{1}^{k-k_{r-1}-\Delta_{r-1}} V_{i}\left(k_{r-1}+\Delta_{r-1}\right)+\sum_{\zeta=k_{r-1}+\Delta_{r-1}}^{k-1} \mu_{1}^{k-1-\varsigma} \Xi(\varsigma), \forall k \in\left[k_{r-1}+\Delta_{r-1}, k_{r}\right), \\
\mu_{2}^{k-k_{r}} V_{(i, j)}\left(k_{r}\right)+\sum_{\zeta=k_{r}}^{k-1} \mu_{2}^{k-1-\varsigma} \Xi(\varsigma), \forall k \in\left[k_{r}, k_{r}+\Delta_{r}\right) .
\end{array}\right. \\
& \text { Noting the condition (29), then } \\
& V_{i}(k) \leq\left\{\begin{array}{l}
\lambda \mu_{1}^{k-k_{r-1}-\Delta_{r-1}} V_{i}\left(k_{r-1}+\Delta_{r-1}\right)+\sum_{\varsigma=k_{r-1}+\Delta_{r-1}}^{k-1} \mu_{1}^{k-1-\varsigma_{\Xi}}(\varsigma), \forall k \in\left[k_{r-1}, k_{r-1}+\Delta_{r-1}\right), \\
\lambda \mu_{2}^{k-k_{r}} V_{(i, j)}\left(k_{r}\right)+\sum_{\zeta=k_{r}}^{k-1} \mu_{2}^{k-1-\varsigma} \Xi(\varsigma), \forall k \in\left[k_{r-1}+\Delta_{r-1}, k_{r}\right) .
\end{array}\right. \\
& \text { For } T \in\left[k_{N_{\sigma}\left(T, k_{0}\right)}+\Delta_{N_{\sigma}\left(T, k_{0}\right)}, k_{\left.N_{\sigma}\left(T, k_{0}\right)+1\right)}\right. \text {, repeating (58) and (59) follows that: } \\
& V_{\sigma\left(k_{\aleph+1}\right)}(T) \leq \mu_{1}^{T-k_{\aleph}-\Delta_{\aleph}} V_{\sigma\left(k_{\aleph+1}\right)}\left(k_{\aleph}+\Delta_{\aleph}\right)+\sum_{\zeta=k_{\aleph}+\Delta_{\aleph}}^{T-1} \mu_{1}^{T-1-\varsigma} \Xi(\varsigma) \\
& \leq \lambda \mu_{1}^{T-k_{\aleph}-\Delta_{\aleph}} \mu_{2}^{\Delta_{\aleph}} V_{\sigma\left(k_{\aleph}+\Delta_{\aleph}\right)}\left(k_{\aleph}\right)+\sum_{\zeta=k_{\aleph}+\Delta_{\aleph}}^{T-1} \mu_{1}^{T-1-\varsigma^{-}} \Xi(\varsigma) \\
& +\lambda \mu_{1}^{T-k_{\aleph}-\Delta_{\aleph}} \sum_{\varsigma=k_{\aleph}}^{k_{\aleph}+\Delta_{\aleph}-1} \mu_{2}^{k_{\aleph}+\Delta_{\aleph}-1-\varsigma} \\
& \leq \lambda^{2} \mu_{1}^{T-k_{\aleph-1}-\Delta_{\aleph}-\Delta_{\aleph-1}} \mu_{2}^{\Delta_{\aleph}} V_{\sigma\left(k_{\aleph-1}\right)}\left(k_{\aleph-1}+\Delta_{\aleph-1}\right)+\sum_{\zeta=k_{\aleph}+\Delta_{\aleph}}^{T-1} \mu_{1}^{T-1-\varsigma_{\Xi}}(\varsigma) \\
& +\lambda \mu_{1}^{T-k_{\aleph}-\Delta_{\aleph}} \sum_{\zeta=k_{\aleph}}^{k_{\aleph}+\Delta_{\aleph}-1} \mu_{2}^{k_{\aleph}+\Delta_{\aleph}-1-\varsigma} \\
& +\lambda^{2} \mu_{2}^{\Delta_{\aleph}} \sum_{\varsigma=k_{\aleph-1}+\Delta_{\aleph-1}}^{k_{\aleph}-1} \mu_{1}^{T-1-\Delta_{\aleph}-\varsigma} \Xi(\varsigma) \\
& =\lambda^{2} e^{\left(T-k_{\aleph-1}-\Delta_{\aleph}-\Delta_{\aleph-1}\right) \ln \mu_{1}} e^{\Delta_{\aleph} \ln \mu_{2}} V_{\sigma\left(k_{\aleph-1}\right)}\left(k_{\aleph-1}+\Delta_{\aleph-1}\right)+\sum_{\zeta=k_{\aleph}+\Delta_{\aleph}}^{T-1} e^{(T-1-\varsigma) \ln \mu_{1}} \Xi(\varsigma) \\
& +\lambda e^{\left(T-k_{\aleph}-\Delta_{\aleph}\right) \ln \mu_{1}} \sum_{\varsigma=k_{\aleph}}^{k_{\aleph}+\Delta_{\aleph}-1} e^{\left(k_{\aleph}+\Delta_{\aleph}-1-\varsigma\right) \ln \mu_{2}} \\
& +\lambda^{2} e^{\Delta_{\aleph} \ln \mu_{2}} \sum_{\varsigma=k_{\aleph-1}+\Delta_{\aleph-1}}^{k_{\aleph}-1} e^{\left(T-1-\Delta_{\aleph}-\varsigma\right) \ln \mu_{1}} \Xi(\varsigma) \\
& \leq \cdots \\
& \leq e^{2 \aleph \ln \lambda} e^{\left(T-k_{0}-\aleph \Delta_{\max }\right) \ln \mu_{1}} e^{\aleph \Delta_{\max } \ln \mu_{2}} V_{\sigma\left(k_{0}\right)}\left(k_{0}\right) \\
& +\sum_{\zeta=k_{0}}^{T-1} e^{2 N_{\sigma}(T, \zeta) \ln \lambda} e^{N_{\sigma}(T-1, \zeta) \Delta_{\max } \ln \mu_{2}} e^{\left(T-1-N_{\sigma}(T-1, \zeta) \Delta_{\max }-\zeta\right) \ln \mu_{1}} \Xi(\varsigma),
\end{aligned}
$$$$
\text { where } \aleph=N_{\sigma}\left(T, k_{0}\right) \text { and } \Delta_{\max }=\max \left\{\Delta_{1}, \Delta_{2}, \ldots, \Delta_{\aleph}\right\} \text {. Under zero initial conditions, }
$$
we have

$$
0 \leq \sum_{\zeta=k_{0}}^{T-1} e^{2 N_{\sigma}(T, \zeta) \ln \lambda} e^{N_{\sigma}(T-1, \zeta) \Delta_{\max } \ln \mu_{2}} e^{\left(T-1-N_{\sigma}(T-1, \zeta) \Delta_{\max }-\varsigma\right) \ln \mu_{1}} \Xi(\varsigma),
$$

that is,

$$
\begin{aligned}
& \sum_{\zeta=k_{0}}^{T-1} e^{2 N_{\sigma}(T, \zeta) \ln \lambda} e^{N_{\sigma}(T-1, \zeta) \Delta_{\max } \ln \mu_{2}} e^{\left(T-1-N_{\sigma}(T-1, \zeta) \Delta_{\max }-\varsigma\right) \ln \mu_{1}}\|e(\zeta)\|_{1} \\
\leq & \gamma \sum_{\zeta=k_{0}}^{T-1} e^{2 N_{\sigma}(T, \zeta) \ln \lambda} e^{N_{\sigma}(T-1, \zeta) \Delta_{\max } \ln \mu_{2}} e^{\left(T-1-N_{\sigma}(T-1, \zeta) \Delta_{\max }-\zeta\right) \ln \mu_{1}}\|\omega(\zeta)\|_{1} .
\end{aligned}
$$

Multiplying both sides of the inequality (62) with $e^{\left(\ln \mu_{1}-\ln \mu_{2}\right) N_{\sigma}\left(T, k_{0}\right) \Delta_{\max }-2 N_{\sigma}\left(T, k_{0}\right) \ln \lambda}$ gives

$$
\begin{gathered}
\sum_{\zeta=k_{0}}^{T-1} e^{(T-1-\varsigma) \ln \mu_{1}+\left(\ln \mu_{1}-\ln \mu_{2}\right) N_{\sigma}(\varsigma, 0) \Delta_{\max }-2 N_{\sigma}(\varsigma, 0) \ln \lambda}\|e(\varsigma)\|_{1} \\
\leq \gamma \sum_{\zeta=k_{0}}^{T-1} e^{(T-1-\varsigma) \ln \mu_{1}+\left(\ln \mu_{1}-\ln \mu_{2}\right) N_{\sigma}(\varsigma, 0) \Delta_{\max }-2 N_{\sigma}(\varsigma, 0) \ln \lambda}\|\omega(\varsigma)\|_{1} .
\end{gathered}
$$

From Definition 2 and (34), it is clear that

$$
N_{\sigma}\left(\varsigma, k_{0}\right) \leq N_{0}+\frac{\left(\varsigma-k_{0}\right) \ln \mu_{1}^{*}}{2 \ln \lambda+\left(\ln \mu_{2}-\ln \mu_{1}\right) \Delta_{\max }} .
$$

Then, (63) can be transformed into

$$
\begin{gathered}
\sum_{\varsigma=k_{0}}^{T-1} e^{(T-1-\varsigma) \ln \mu_{1}-\left[2 \ln \lambda+\left(\ln \mu_{2}-\ln \mu_{1}\right) \Delta_{\max }\right]\left(N_{0}+\frac{\left(\varsigma-k_{0}\right) \ln \mu_{1}^{*}}{2 \ln \lambda+\left(\ln \mu_{2}-\ln \mu_{1}\right) \Delta \max }\right)}\|e(\varsigma)\|_{1} \\
\leq \gamma \sum_{\zeta=k_{0}}^{T-1} e^{(T-1-\varsigma) \ln \mu_{1}-\left[2 \ln \lambda+\left(\ln \mu_{2}-\ln \mu_{1}\right) \Delta_{\max }\right]\left(N_{0}+\frac{\left(\varsigma-k_{0}\right) \ln \mu_{1}^{*}}{2 \ln \lambda+\left(\ln \mu_{2}-\ln \mu_{1}\right) \Delta \max }\right)}\|\omega(\varsigma)\|_{1} .
\end{gathered}
$$


The above inequality can be further written as

$$
\begin{aligned}
& \sum_{\zeta=k_{0}}^{T-1} e^{(T-1) \ln \mu_{1}-k_{0} \ln \mu_{1}^{*}} e^{\left(\ln \mu_{1}^{*}-\ln \mu_{1}\right) \varsigma}\|e(\varsigma)\|_{1} \\
\leq & \gamma \sum_{\zeta=k_{0}}^{T-1} e^{(T-1) \ln \mu_{1}-k_{0} \ln \mu_{1}^{*}} e^{\left(\ln \mu_{1}^{*}-\ln \mu_{1}\right) \zeta}\|\omega(\varsigma)\|_{1} .
\end{aligned}
$$

From (66), we can obtain

$$
\sum_{\zeta=k_{0}}^{T-1} e^{-\left(\ln \mu_{1}^{*}-\ln \mu_{1}\right) \varsigma}\|e(\varsigma)\|_{1} \leq \gamma \sum_{\zeta=k_{0}}^{T-1}\|\omega(\varsigma)\|_{1} .
$$

Summing from 0 to $\infty$ for both sides of (67), it yields that

$$
\sum_{k=0}^{\infty} e^{-\hbar k}\|e(k)\|_{1} \leq \gamma \sum_{k=0}^{\infty}\|\omega(k)\|_{1},
$$

where $\hbar=\ln \mu_{1}^{*}-\ln \mu_{1}$. By Definition 3, the error systems (9) and (10) satisfy the $\ell_{1}$-gain performance index (68).

Remark 4. Generally, the signal can be quantized during the actual transmission process due to various reasons, such as energy consumption issues, intermittent sensor fault, limited digital communication resource, and so on. It is necessary to incorporate the quantization technique with event-triggering mechanism to generate the quantized output. Theorem 1 first introduces the quantization technique to the filter design of positive systems, where a sector restriction is adopted to analyze and mitigate the quantization effect [47-49]. Currently, few efforts have been devoted to positive systems, though the quantization approach is effective and practical for dealing with many practical problems. It is interesting to develop the quantization approach in Theorem 1 for other issues of positive systems.

\subsection{Dynamic Event-Triggering Case}

In this section, we propose a dynamic event-triggering mechanism as an alternative of the static event-triggering mechanism for system (1). Define the sampling error of the event generator as $e_{y}(k)=\widetilde{y}(k)-y(k)$, where $\widetilde{y}(k)=y\left(k_{\wp}\right), y\left(k_{\wp}\right)$ is the output signal of the event generator at the event-triggering instant $k_{\wp}, \wp \in \mathbb{N}$. Following this, the output will be released by the following dynamic event-triggering condition:

$$
\left\|e_{y}(k)\right\|_{1}>\beta\|y(k)\|_{1}+\frac{1}{\psi} \eta(k) \vee \eta(k)>\|y(k)\|_{1}
$$

where $\beta$ and $\psi$ are given positive constants, and $\eta(k)$ is an internal dynamic variable satisfying

$$
\eta(k+1)=\varrho \eta(k)+\beta\|y(k)\|_{1}-\left\|e_{y}(k)\right\|_{1},
$$

with $\eta\left(k_{0}\right)=\eta_{0}$ as the initial value and $\varrho \in(0,1)$ as a given constant.

Theorem 2. If there exist constants $0<\omega_{1} \leq \omega_{2}, 0<\omega_{3} \leq \omega_{4}, 0<\omega_{5} \leq \omega_{6}, 0<\varepsilon_{1} \leq \varepsilon_{2}$, $0<\varepsilon_{3} \leq \varepsilon_{4}, 0<\varepsilon_{5} \leq \varepsilon_{6}, 0<\vartheta_{1} \leq \vartheta_{2}, \psi>0, \gamma>0, \lambda>1,0 \leq \beta<1,0<\mu_{1}<1, \mu_{2}>1$, $\mathbb{R}^{n}$ vectors $\zeta_{i} \succ 0, \zeta_{(i, j)} \succ 0, \varphi_{i} \succ 0, \varphi_{(i, j)} \succ 0, \xi_{i} \succeq 0, \xi_{i \imath} \succeq 0, \xi_{j} \succeq 0, \xi_{j l} \succeq 0, \rho_{i j} \succeq 0$, and $\mathbb{R}^{m}$ vectors $\delta_{i} \succeq 0, \delta_{i \imath} \succeq 0, \delta_{j} \succeq 0, \delta_{j l} \succeq 0, \theta_{i j} \succeq 0$ such that

$$
\begin{gathered}
\vartheta_{1} \sum_{l=1}^{n} 1_{n}^{(\imath)} \xi_{i l}^{\top}+\omega_{3} \sum_{l=1}^{n} 1_{n}^{(l)} \delta_{i l}^{\top} L \Psi C_{i}-\omega_{2} 1_{n}^{\top} \varphi_{i} A_{i} \succeq 0, \\
\varepsilon_{3} \sum_{l=1}^{n} 1_{n}^{(l)} \delta_{i l}^{\top} L \Psi D_{i}-\varepsilon_{2} 1_{n}^{\top} \varphi_{i} B_{i} \succeq 0, \\
\omega_{5} \sum_{\jmath=1}^{s} 1_{s}^{(\jmath)} \rho_{i \jmath}^{\top}+\omega_{3} \sum_{\jmath=1}^{s} 1_{s}^{(\jmath)} \theta_{i j}^{\top} L \Psi C_{i}-\omega_{6} E_{i} \succeq 0, \\
\varepsilon_{3} \sum_{\jmath=1}^{s} 1_{s}^{(\jmath)} \theta_{i j}^{\top} L \Psi D_{i}-\varepsilon_{6} F_{i} \succeq 0, \\
\vartheta_{1} \sum_{l=1}^{n} 1_{n}^{(l)} \xi_{j l}^{\top}+\omega_{3} \sum_{l=1}^{n} 1_{n}^{(l)} \delta_{j l}^{\top} L \Psi C_{i}-\omega_{2} 1_{n}^{\top} \varphi_{j} A_{i} \succeq 0,
\end{gathered}
$$




$$
\begin{aligned}
& \varepsilon_{3} \sum_{l=1}^{n} 1_{n}^{(l)} \delta_{j l}^{\top} L \Psi D_{i}-\varepsilon_{2} 1_{n}^{\top} \varphi_{j} B_{i} \succeq 0, \\
& \omega_{5} \sum_{\jmath=1}^{s} 1_{s}^{(\jmath)} \rho_{j \jmath}^{\top}+\omega_{3} \sum_{\jmath=1}^{s} 1_{s}^{(\jmath)} \theta_{j \jmath}^{\top} L \Psi C_{i}-\omega_{6} E_{i} \succeq 0, \\
& \varepsilon_{3} \sum_{\jmath=1}^{S} 1_{s}^{(\jmath)} \theta_{j \jmath}^{\top} L \Psi D_{i}-\varepsilon_{6} F_{i} \succeq 0, \\
& \omega_{2} A_{i}^{\top} \zeta_{i}+\vartheta_{2} \xi_{i}+\omega_{4} C_{i}^{\top} \Phi J \delta_{i}-\omega_{1} A_{i}^{\top} \varphi_{i}-\mu_{1} \zeta_{i}+\left(\omega_{6} \sum_{\jmath=1}^{s} 1_{s}^{(j)} \rho_{i j}^{\top}\right. \\
& \left.+\mathfrak{\omega}_{4} \sum_{\jmath=1}^{s} 1_{s}^{(\jmath)} \theta_{i j}^{\top} J \Phi C_{i}-\omega_{5} E_{i}\right)^{\top} 1_{s}+\beta \omega_{4} C_{i}^{\top} 1_{m} \preceq 0, \\
& \omega_{6}\left(\sum_{\jmath=1}^{s} 1_{s}^{(\jmath)} \rho_{i j}^{\top}\right)^{\top} 1_{s}+\vartheta_{2} \xi_{i}-\mu_{1} \varphi_{i} \preceq 0, \\
& \varepsilon_{2} B_{i}^{\top} \zeta_{i}+\beta \varepsilon_{4} D_{i}^{\top} 1_{m}+\varepsilon_{4} D_{i}^{\top} \Phi J \delta_{i}-\varepsilon_{1} B_{i}^{\top} \varphi_{i}+\varepsilon_{4} D_{i}^{\top} \Phi J\left(\sum_{\jmath=1}^{s} 1_{s}^{(\jmath)} \theta_{i j}^{\top}\right)^{\top} 1_{s} \\
& -\varepsilon_{5} F_{i}^{\top} 1_{s}-\gamma 1_{m} \preceq 0 \text {, } \\
& \omega_{2} A_{i}^{\top} \zeta_{(i, j)}+\vartheta_{2} \xi_{j}+\omega_{4} C_{i}^{\top} \Phi J \delta_{j}-\omega_{1} A_{i}^{\top} \varphi_{(i, j)}-\mu_{2} \zeta_{(i, j)}+\left(\omega_{6} \sum_{j=1}^{s} 1_{s}^{(j)} \rho_{j j}^{\top}\right. \\
& \left.+\omega_{4} \sum_{\jmath=1}^{s} 1_{s}^{(\jmath)} \theta_{j \jmath}^{\top} J \Phi C_{i}-\omega_{5} E_{i}\right)^{\top} 1_{s}+\beta \omega_{4} C_{i}^{\top} 1_{m} \preceq 0, \\
& \omega_{6}\left(\sum_{\jmath=1}^{s} 1_{s}^{(\jmath)} \rho_{j j}^{\top}\right)^{\top} 1_{s}+\vartheta_{2} \xi_{j}-\mu_{2} \varphi_{(i, j)} \preceq 0, \\
& \varepsilon_{2} B_{i}^{\top} \zeta_{(i, j)}+\beta \varepsilon_{4} D_{i}^{\top} 1_{m}+\varepsilon_{4} D_{i}^{\top} \Phi J \delta_{j}-\varepsilon_{1} B_{i}^{\top} \varphi_{(i, j)}+\varepsilon_{4} D_{i}^{\top} \Phi J\left(\sum_{\jmath=1}^{s} 1_{s}^{(j)} \theta_{j \jmath}^{\top}\right)^{\top} 1_{s} \\
& -\varepsilon_{5} F_{i}^{\top} 1_{s}-\gamma 1_{m} \preceq 0 \text {, } \\
& \zeta_{i} \preceq \lambda \zeta_{(i, j)}, \zeta_{i} \preceq \lambda \zeta_{(j, i)}, \zeta_{(i, j)} \preceq \lambda \zeta_{i}, \zeta_{(j, i)} \preceq \lambda \zeta_{i}, \\
& \varphi_{i} \preceq \lambda \varphi_{(i, j)}, \varphi_{i} \preceq \lambda \varphi_{(j, i)}, \varphi_{(i, j)} \preceq \lambda \varphi_{i}, \varphi_{(j, i)} \preceq \lambda \varphi_{i}, \\
& \xi_{i l} \preceq \xi_{i}, \delta_{i l} \preceq \delta_{i}, \xi_{j l} \preceq \xi_{j}, \delta_{j l} \preceq \delta_{j},
\end{aligned}
$$

hold $\forall i, j \in S, i \neq j, \imath=1,2, \cdots, n$ and $\jmath=1,2, \cdots, s$, then the error systems (9) and (10) are positive and stable with filter gain matrices (31) and (32) and the switching law satisfying

$$
\begin{aligned}
& \frac{\Gamma^{-}\left(k_{0}, k\right)}{\Gamma^{+}\left(k_{0}, k\right)} \geq \frac{\ln \mu_{2}-\ln \mu_{1}}{\ln \mu_{1}^{*}-\ln \mu_{1}}, \mu_{1}^{*} \in\left(\mu_{1}, 1\right), \\
& \tau_{a} \geq \tau_{a}^{*}=-\frac{2 \ln \lambda+\left(\ln \mu_{2}-\ln \mu_{1}\right) \Delta_{\max }}{\ln \mu_{1}^{*}},
\end{aligned}
$$

where $\Psi=I-\left(\beta+\frac{1}{\psi}\right) 1_{m \times m}, \Phi=I+\left(\beta+\frac{1}{\psi}\right) 1_{m \times m}$, and $\Delta_{\text {max }}$ denotes the maximum of time $\operatorname{lag} \Delta_{r}$.

Proof. First, the positivity of the error systems (9) and (10) are considered. For $x\left(k_{0}\right) \succeq 0$, the output satisfies $y\left(k_{0}\right) \succeq 0$. We can obtain from the dynamic event-triggering condition (69) and (70) that

$$
\left\|e_{y}\left(k_{0}\right)\right\|_{1} \leq \beta\left\|y\left(k_{0}\right)\right\|_{1}+\frac{1}{\psi} \eta\left(k_{0}\right) \leq\left(\beta+\frac{1}{\psi}\right) \mathbf{1}_{m}^{\top} y\left(k_{0}\right),
$$

which leads to

$$
-\left(\beta+\frac{1}{\psi}\right) \mathbf{1}_{m \times m} y\left(k_{0}\right) \preceq e_{y}\left(k_{0}\right) \preceq\left(\beta+\frac{1}{\psi}\right) \mathbf{1}_{m \times m} y\left(k_{0}\right) .
$$

From (11), (13), and (90), it is clear that, for $k \in\left[k_{r}, k_{r}+\Delta_{r}\right)$,

$$
\begin{gathered}
\widetilde{x}\left(k_{0}+1\right) \\
e\left(k_{0}\right) \succeq \widetilde{\widetilde{A}}_{2 i j} \widetilde{\widetilde{x}}\left(k_{0}\right)+\underline{\widetilde{B}}_{2 i j} \widetilde{x}\left(k_{0}\right)+\underline{\widetilde{F}}_{2 i j} \omega\left(k_{0}\right),
\end{gathered}
$$


where

$$
\begin{aligned}
& \underline{\widetilde{A}}_{2 i j}=\left(\begin{array}{cc}
\omega_{1} A_{i} & 0 \\
\vartheta_{1} A_{f j}+\omega_{3} B_{f j} L \Psi C_{i}-\omega_{2} A_{i} & \vartheta_{1} A_{f j}
\end{array}\right), \underline{\widetilde{B}}_{2 i j}=\left(\begin{array}{c}
\varepsilon_{1} B_{i} \\
\varepsilon_{3} B_{f j} L \Psi D_{i}-\varepsilon_{2} B_{i}
\end{array}\right), \\
& \widetilde{\underline{E}}_{2 i j}=\left(\omega_{5} E_{f j}+\omega_{3} F_{f j} L \Psi C_{i}-\omega_{6} E_{i} \quad \omega_{5} E_{f j}\right), \underline{\widetilde{F}}_{2 i j}=\left(\varepsilon_{3} F_{f j} L \Psi D_{i}-\varepsilon_{6} F_{i}\right) \text {. }
\end{aligned}
$$

and for $k \in\left[k_{r}+\Delta_{r}, k_{r+1}\right)$,

$$
\begin{gathered}
\widetilde{x}\left(k_{0}+1\right) \succeq \widetilde{A}_{2 i} \widetilde{x}\left(k_{0}\right)+\widetilde{\widetilde{B}}_{2 i} \omega\left(k_{0}\right), \\
e\left(k_{0}\right) \succeq \underline{\widetilde{E}}_{2 i} \widetilde{x}\left(k_{0}\right)+\underline{\widetilde{F}}_{2 i} \omega\left(k_{0}\right),
\end{gathered}
$$

where

$$
\begin{aligned}
& \underline{\widetilde{A}}_{2 i}=\left(\begin{array}{cc}
\omega_{1} A_{i} & 0 \\
\vartheta_{1} A_{f i}+\omega_{3} B_{f i} L \Psi C_{i}-\omega_{2} A_{i} & \vartheta_{1} A_{f i}
\end{array}\right), \underline{\underline{B}}_{2 i}=\left(\begin{array}{c}
\varepsilon_{1} B_{i} \\
\varepsilon_{3} B_{f i} L \Psi D_{i}-\varepsilon_{2} B_{i}
\end{array}\right), \\
& \widetilde{E}_{2 i}=\left(\omega_{5} E_{f i}+\omega_{3} F_{f i} L \Psi C_{i}-\omega_{6} E_{i} \quad \omega_{5} E_{f i}\right), \widetilde{\underline{F}}_{2 i}=\left(\varepsilon_{3} F_{f i} L \Psi D_{i}-\varepsilon_{6} F_{i}\right) .
\end{aligned}
$$

Using a similar method in Theorem 1 gives that the error systems (9) and (10) are positive. From (12) and (90), for $k \in\left[k_{r}, k_{r}+\Delta_{r}\right)$, we have

$$
\begin{gathered}
\widetilde{x}(k+1) \preceq \overline{\widetilde{A}}_{2 i j} \widetilde{x}(k)+\overline{\widetilde{B}}_{2 i j} \omega(k), \\
e(k) \preceq \widetilde{\widetilde{E}}_{2 i j} \widetilde{x}(k)+\widetilde{\widetilde{F}}_{2 i j} \omega(k),
\end{gathered}
$$

where

$$
\begin{gathered}
\overline{\widetilde{A}}_{2 i j}=\left(\begin{array}{cc}
\omega_{2} A_{i} & 0 \\
\vartheta_{2} A_{f j}+\omega_{4} B_{f j} J \Phi C_{i}-\omega_{1} A_{i} & \vartheta_{2} A_{f j}
\end{array}\right), \overline{\widetilde{B}}_{2 i j}=\left(\begin{array}{c}
\varepsilon_{2} B_{i} \\
\varepsilon_{4} B_{f j} J \Phi D_{i}-\varepsilon_{1} B_{i}
\end{array}\right), \\
\widetilde{\widetilde{E}}_{2 i j}=\left(\begin{array}{ll}
\omega_{6} E_{f j}+\omega_{4} F_{f j} J \Phi C_{i}-\omega_{5} E_{i} & \omega_{6} E_{f j}
\end{array}\right), \widetilde{\widetilde{F}}_{2 i j}=\left(\varepsilon_{4} F_{f j} J \Phi D_{i}-\varepsilon_{5} F_{i}\right) .
\end{gathered}
$$

For $k \in\left[k_{r}+\Delta_{r}, k_{r+1}\right)$, we have

$$
\begin{gathered}
\widetilde{x}(k+1) \preceq \overline{\widetilde{A}}_{2 i} \widetilde{x}(k)+\overline{\widetilde{B}}_{2 i} \omega(k), \\
e(k) \preceq \widetilde{\widetilde{E}}_{2 i} \widetilde{x}(k)+\widetilde{\widetilde{F}}_{2 i} \omega(k),
\end{gathered}
$$

where

$$
\begin{aligned}
& \widetilde{\widetilde{A}}_{2 i}=\left(\begin{array}{cc}
\omega_{2} A_{i} & 0 \\
\vartheta_{2} A_{f i}+\omega_{4} B_{f i} J \Phi C_{i}-\omega_{1} A_{i} & \vartheta_{2} A_{f i}
\end{array}\right), \widetilde{\widetilde{B}}_{2 i}=\left(\begin{array}{c}
\varepsilon_{2} B_{i} \\
\varepsilon_{4} B_{f i} J \Phi D_{i}-\varepsilon_{1} B_{i}
\end{array}\right), \\
& \widetilde{\widetilde{E}}_{2 i}=\left(\omega_{6} E_{f i}+\omega_{4} F_{f i} J \Phi C_{i}-\omega_{5} E_{i} \quad \omega_{6} E_{f i}\right), \widetilde{\widetilde{F}}_{2 i}=\left(\varepsilon_{4} F_{f i} J \Phi D_{i}-\varepsilon_{5} F_{i}\right) .
\end{aligned}
$$

Choose a linear copositive Lyapunov function:

$$
V_{i}(k)=\left\{\begin{array}{l}
\widetilde{x}^{\top}(k) v_{i}+\eta(k), \forall k \in\left[k_{r-1}+\Delta_{r-1}, k_{r}\right), \\
\tilde{x}^{\top}(k) v_{(i, j)}+\eta(k), \forall k \in\left[k_{r}, k_{r}+\Delta_{r}\right) .
\end{array}\right.
$$

Combining (93) and (94), we find

$$
\Delta V_{i}(k) \leq\left\{\begin{array}{l}
\tilde{x}^{\top}(k) \Omega_{1}+\omega^{\top}(k) \Gamma_{1}+(\varrho-1) \eta(k), \forall k \in\left[k_{r-1}+\Delta_{r-1}, k_{r}\right), \\
\widetilde{x}^{\top}(k) \Omega_{2}+\omega^{\top}(k) \Gamma_{2}+(\varrho-1) \eta(k), \forall k \in\left[k_{r}, k_{r}+\Delta_{r}\right),
\end{array}\right.
$$

where 


$$
\begin{gathered}
\Omega_{1}=\overline{\widetilde{A}}_{2 i}^{\top} v_{i}-v_{i}=\left(\begin{array}{c}
\omega_{2} A_{i}^{\top} \zeta_{i}+\left(\vartheta_{2} A_{f i}^{\top}+\omega_{4} C_{i}^{\top} \Phi J B_{f i}^{\top}-\omega_{1} A_{i}^{\top}\right) \varphi_{i}+\beta \omega_{4} C_{i}^{\top} 1_{m}-\zeta_{i} \\
\vartheta_{2} A_{f i}^{\top} \varphi_{i}-\varphi_{i}
\end{array}\right), \\
\Omega_{2}=\widetilde{\widetilde{A}}_{2 i j}^{\top} v_{(i, j)}-v_{(i, j)}=\left(\begin{array}{c}
\left.\omega_{2} A_{i}^{\top} \zeta_{(i, j)}+\left(\vartheta_{2} A_{f j}^{\top}+\omega_{4} C_{i}^{\top} \Phi J B_{f j}^{\top}-\omega_{1} A_{i}^{\top}\right) \varphi_{(i, j)}+\beta \omega_{4} C_{i}^{\top} 1_{m}-\zeta_{(i, j)}\right) \\
\vartheta_{2} A_{f j}^{\top} \varphi_{(i, j)}-\varphi_{(i, j)}
\end{array}\right), \\
\Gamma_{1}=\overline{\widetilde{B}}_{2 i}^{\top} v_{i}=\varepsilon_{2} B_{i}^{\top} \zeta_{i}+\beta \varepsilon_{4} D_{i}^{\top} 1_{m}+\left(\varepsilon_{4} D_{i}^{\top} \Phi J B_{f i}^{\top}-\varepsilon_{1} B_{i}^{\top}\right) \varphi_{i}, \\
\Gamma_{2}=\widetilde{\widetilde{B}}_{2 i j}^{\top} v_{(i, j)}=\varepsilon_{2} B_{i}^{\top} \zeta_{(i, j)}+\beta \varepsilon_{4} D_{i}^{\top} 1_{m}+\left(\varepsilon_{4} D_{i}^{\top} \Phi J B_{f j}^{\top}-\varepsilon_{1} B_{i}^{\top}\right) \varphi_{(i, j)} .
\end{gathered}
$$

Using a similar method to Theorem 1 , it is not difficult to obtain (68) under average dwell time (88), which means that the error systems (9) and (10) are $\ell_{1}$-gain stable with performance $\gamma$.

Remark 5. Compared with the existing results on static event-triggering strategies of positive systems [39-41], the dynamic strategy proposed in Theorem 2 is more flexible and releases fewer data. For the dynamic event-triggered issues of general systems [42-46], it is clear that dynamic event-triggering conditions cannot be directly used for positive systems. Therefore, we proposed a dynamic event-triggering condition (69), and the lower bound of the error systems can be obtained from an interval system. Based on this point, the dynamic event-triggering condition can be applied to other issues of positive systems, such as output feedback control, observer design, etc.

\section{Illustrative Examples}

Two examples are provided to verify the effectiveness of the proposed design.

Example 1. Consider the system (1) with two subsystems:

$$
\begin{aligned}
& A_{1}=\left(\begin{array}{lll}
0.1503 & 0.1452 & 0.0231 \\
0.2046 & 0.1729 & 0.1967 \\
0.1050 & 0.2130 & 0.1714
\end{array}\right), B_{1}=\left(\begin{array}{ll}
0.0519 & 0.2034 \\
0.1107 & 0.0257 \\
0.0173 & 0.1322
\end{array}\right), C_{1}=\left(\begin{array}{ccc}
0.3 & 0.3 & 0.2 \\
0.4 & 0.1 & 0.1
\end{array}\right) \text {, } \\
& D_{1}=\left(\begin{array}{ll}
0.3 & 0.2 \\
0.2 & 0.4
\end{array}\right), E_{1}=\left(\begin{array}{lll}
0.1 & 0.2 & 0.2
\end{array}\right), F_{1}=\left(\begin{array}{ll}
0.3 & 0.6
\end{array}\right), \\
& A_{2}=\left(\begin{array}{lll}
0.2214 & 0.0496 & 0.1183 \\
0.1426 & 0.1651 & 0.1742 \\
0.1357 & 0.2073 & 0.0137
\end{array}\right), B_{2}=\left(\begin{array}{ll}
0.2641 & 0.2026 \\
0.1273 & 0.1797 \\
0.2065 & 0.1842
\end{array}\right), C_{2}=\left(\begin{array}{lll}
0.2 & 0.4 & 0.2 \\
0.2 & 0.3 & 0.1
\end{array}\right) \text {, } \\
& D_{2}=\left(\begin{array}{ll}
0.2 & 0.3 \\
0.1 & 0.2
\end{array}\right), E_{2}=\left(\begin{array}{lll}
0.1 & 0.2 & 0.2
\end{array}\right), F_{2}=\left(\begin{array}{ll}
0.4 & 0.5
\end{array}\right),
\end{aligned}
$$

where $f_{i}\left(x_{i}(k)\right)=2 e^{-0.2 k} x_{i}(k), \hat{f}_{i}\left(x_{i}(k)\right)=e^{-k} x_{f i}(k), h_{i}\left(x_{i}(k)\right)=x_{i}(k)+\frac{x_{i}(k)}{x_{i}^{2}(k)+1}, p_{i}\left(x_{i}(k)\right)=$ $x_{i}(k)+\frac{x_{i}(k)}{x_{i}^{2}(k)+5}$, the disturbance signal is $\omega_{l}(k)=\left(\begin{array}{ll}\frac{0.4}{(k+1)^{2 / 3}} & 0.4 e^{-0.1 k}\end{array}\right)^{\top}$, and the nonlinear disturbance is given as $g_{l}\left(\omega_{l}(k)\right)=0.3 e^{-0.03 k} \omega_{l}(k), l_{l}\left(\omega_{l}(k)\right)=\omega_{l}(k), q_{l}\left(\omega_{l}(k)\right)=0.75 \omega_{l}(k)$. Then, $\omega_{1}=0.20, \omega_{2}=0.30, \omega_{3}=0.30, \omega_{4}=0.50, \omega_{5}=0.20, \omega_{6}=0.30, \varepsilon_{1}=0.10, \varepsilon_{2}=0.20$, $\varepsilon_{3}=1, \varepsilon_{4}=1, \varepsilon_{5}=0.30, \varepsilon_{6}=0.50$. Choose $\mu_{1}=0.69, \mu_{1}^{*}=0.80, \mu_{2}=1.30, \beta=0.15$, and $\lambda=1.20$. By Theorem 1, the filter gain matrices are:

$$
\begin{aligned}
& A_{f 1}=\left(\begin{array}{lll}
0.3132 & 0.1982 & 0.2287 \\
0.2897 & 0.2154 & 0.2727 \\
0.2698 & 0.2372 & 0.2524
\end{array}\right), B_{f 1}=\left(\begin{array}{ll}
0.5950 & 0.0317 \\
0.5807 & 0.0258 \\
0.5845 & 0.0259
\end{array}\right), E_{f 1}=\left(\begin{array}{l}
0.0013 \\
0.0014 \\
0.0190
\end{array}\right)^{\top}, F_{f 1}=\left(\begin{array}{l}
1.2703 \\
0.9366
\end{array}\right)^{\top}, \\
& A_{f 2}=\left(\begin{array}{lll}
0.1622 & 0.0921 & 0.2278 \\
0.1468 & 0.0894 & 0.2468 \\
0.1493 & 0.0946 & 0.2309
\end{array}\right), B_{f 2}=\left(\begin{array}{ll}
1.3793 & 1.5699 \\
1.5275 & 1.4030 \\
1.5288 & 1.3936
\end{array}\right), E_{f 2}=\left(\begin{array}{l}
0.0055 \\
0.0046 \\
0.0126
\end{array}\right)^{\top}, F_{f 2}=\left(\begin{array}{l}
1.4756 \\
0.6147
\end{array}\right)^{\top},
\end{aligned}
$$

and the $\ell_{1}$-gain value is $\gamma=0.9644$ and average dwell time switching satisfies $\tau_{a} \geq 4.4728$. Figures 2 and 3 denote the event-triggered output signal $\tilde{y}(k)$ and the quantified output signal $\bar{y}(k)$ for nonlinear switched positive systems. The simulations of the output signal $\tilde{y}(k)$ and $\bar{y}(k)$ 
under different initial conditions are shown in Figure 4. Figure 5 provides the simulations of the output $z(k)$ and the estimated output $z_{f}(k)$ under the asynchronous switching signal. Figure 6 shows the event-triggering release interval. The simulations of $z(k)$ and $z_{f}(k)$ under different initial conditions are given in Figure 7.

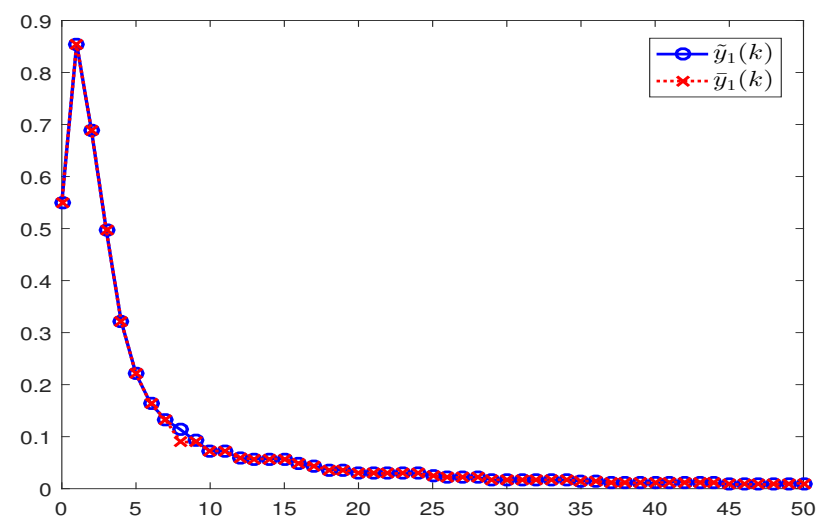

Figure 2. Event-triggered output signal $\tilde{y}_{1}(k)$ and quantified output signal $\bar{y}_{1}(k)$.

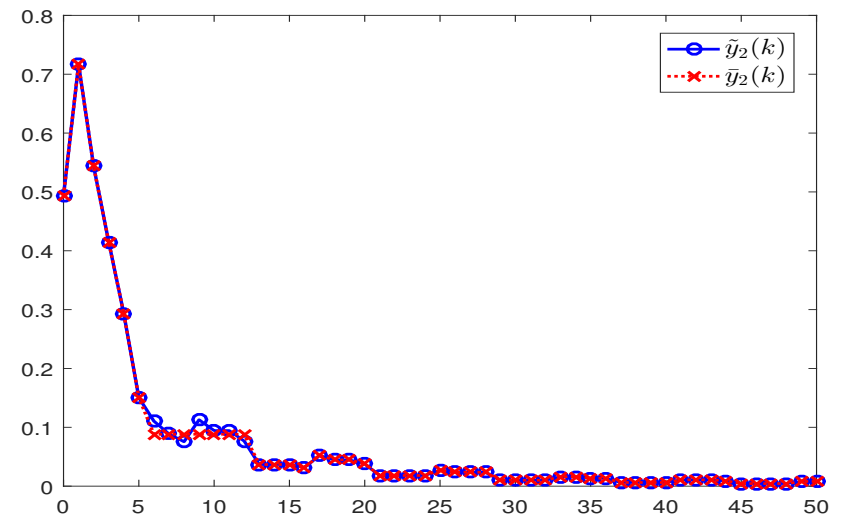

Figure 3. Event-triggered output signal $\tilde{y}_{2}(k)$ and quantified output signal $\bar{y}_{2}(k)$.

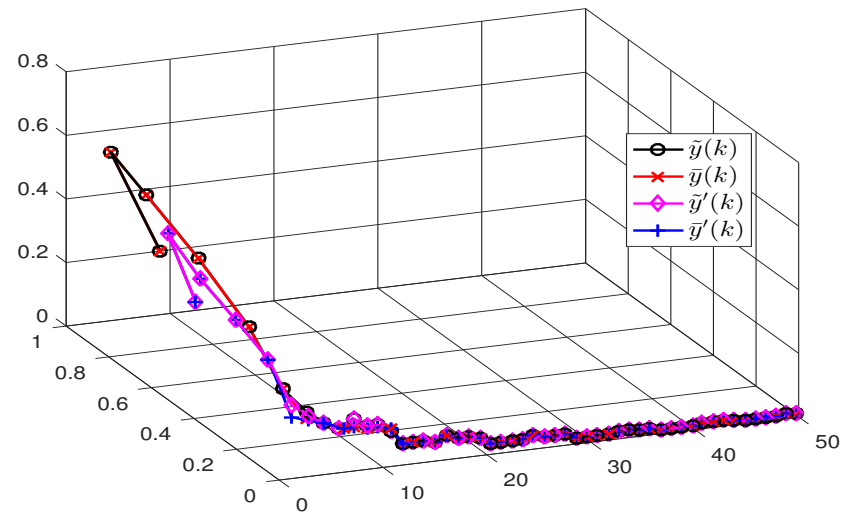

Figure 4. The simulations of $\tilde{y}(k)$ and $\bar{y}(k)$ under different initial conditions. 


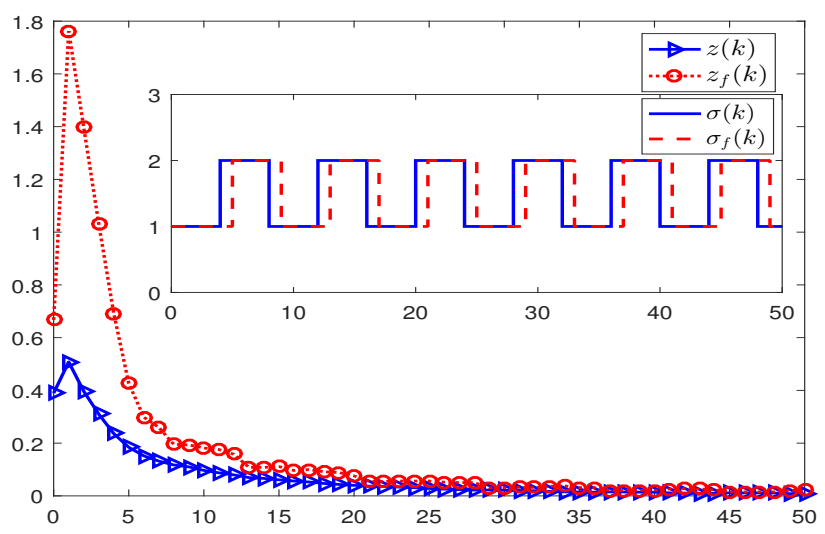

Figure 5. The simulations of $z(k)$ and $z_{f}(k)$ with an asynchronous switching signal.

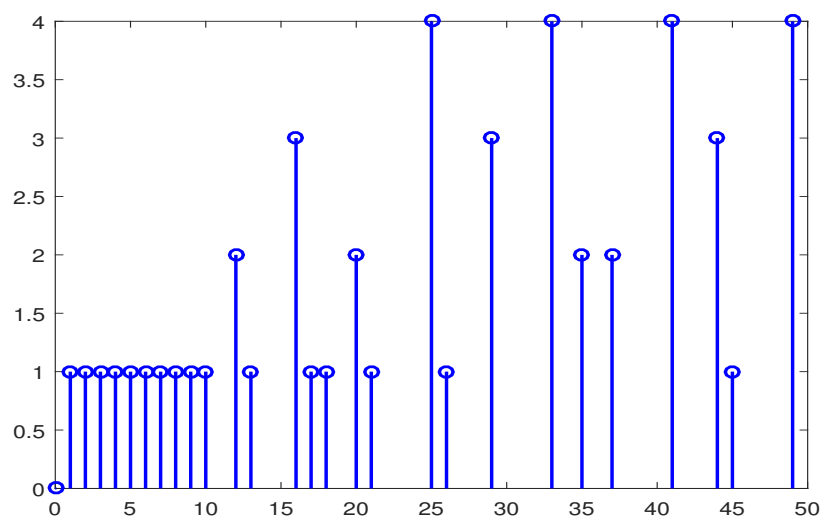

Figure 6. The event-triggering release instants and release intervals.

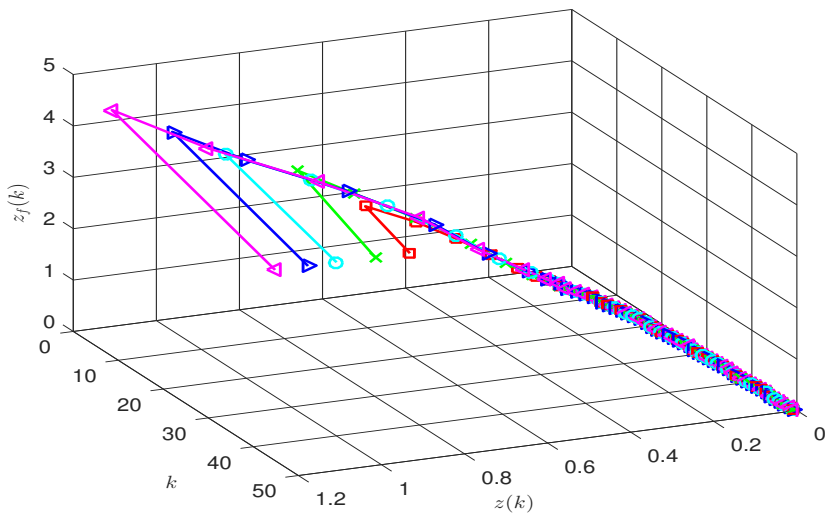

Figure 7. The simulations of $z(k)$ and $z_{f}(k)$ under different initial conditions.

Example 2. Consider the system (1) with two subsystems:

$$
\begin{aligned}
& A_{1}=\left(\begin{array}{lll}
0.0894 & 0.1462 & 0.2763 \\
0.1945 & 0.1573 & 0.2645 \\
0.1497 & 0.0154 & 0.1637
\end{array}\right), B_{1}=\left(\begin{array}{ll}
0.1361 & 0.1104 \\
0.1476 & 0.0632 \\
0.0248 & 0.1353
\end{array}\right), C_{1}=\left(\begin{array}{lll}
0.2 & 0.2 & 0.3 \\
0.3 & 0.2 & 0.1
\end{array}\right) \text {, } \\
& D_{1}=\left(\begin{array}{ll}
0.3 & 0.2 \\
0.2 & 0.4
\end{array}\right), E_{1}=\left(\begin{array}{lll}
0.1 & 0.1 & 0.2
\end{array}\right), F_{1}=\left(\begin{array}{ll}
0.4 & 0.5
\end{array}\right), \\
& A_{2}=\left(\begin{array}{lll}
0.1523 & 0.0496 & 0.1346 \\
0.1817 & 0.1977 & 0.0412 \\
0.1264 & 0.0741 & 0.1255
\end{array}\right), B_{2}=\left(\begin{array}{ll}
0.1450 & 0.2144 \\
0.2145 & 0.1562 \\
0.1855 & 0.1786
\end{array}\right), C_{2}=\left(\begin{array}{lll}
0.3 & 0.1 & 0.1 \\
0.1 & 0.3 & 0.2
\end{array}\right) \text {, } \\
& D_{2}=\left(\begin{array}{ll}
0.3 & 0.3 \\
0.2 & 0.1
\end{array}\right), E_{2}=\left(\begin{array}{lll}
0.2 & 0.1 & 0.2
\end{array}\right), F_{2}=\left(\begin{array}{ll}
0.3 & 0.3
\end{array}\right) .
\end{aligned}
$$


Choose the same parameters as in Example 1. Furthermore, under dynamic event-triggering condition (41) and (42), we give $\eta_{0}=0.60, \varrho=0.50$. By Theorem 2 , the filter gain matrices can be obtained:

$$
\begin{aligned}
& A_{f 1}=\left(\begin{array}{lll}
0.2388 & 0.2588 & 0.3569 \\
0.2685 & 0.2962 & 0.3488 \\
0.2454 & 0.2393 & 0.3122
\end{array}\right), B_{f 1}=\left(\begin{array}{ll}
0.2952 & 0.0460 \\
0.2951 & 0.0451 \\
0.3005 & 0.0550
\end{array}\right), E_{f 1}=\left(\begin{array}{l}
0.0039 \\
0.0034 \\
0.1127
\end{array}\right)^{\top}, F_{f 1}=\left(\begin{array}{l}
1.3585 \\
0.8493
\end{array}\right)^{\top}, \\
& A_{f 2}=\left(\begin{array}{lll}
0.1447 & 0.1751 & 0.2787 \\
0.1513 & 0.1986 & 0.2497 \\
0.1386 & 0.1620 & 0.2379
\end{array}\right), B_{f 2}=\left(\begin{array}{ll}
0.6693 & 0.7391 \\
0.9234 & 0.6583 \\
0.8987 & 0.6241
\end{array}\right), E_{f 2}=\left(\begin{array}{l}
0.0660 \\
0.0385 \\
0.1896
\end{array}\right)^{\top}, F_{f 2}=\left(\begin{array}{l}
0.8772 \\
0.5213
\end{array}\right)^{\top},
\end{aligned}
$$

and the $\ell_{1}$-gain value is $\gamma=1.0796$ and average dwell time switching satisfies $\tau_{a} \geq 4.4728$. The dynamic event-triggered output signal $\tilde{y}(k)$ and the quantified output signal $\bar{y}(k)$ are given in Figures 8 and 9. Figure 10 shows the simulation results of the output $z(k)$ and the estimated output $z_{f}(k)$ under the asynchronous switching signal. Figure 11 shows the dynamic event-triggering release interval. The simulation results of $z(k)$ and $z_{f}(k)$ under different initial conditions are shown in Figure 12.

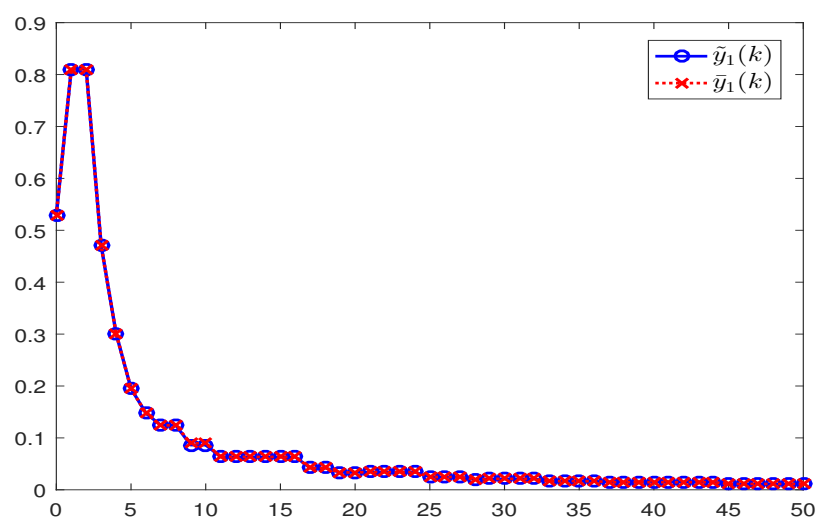

Figure 8. The dynamic event-triggered output signal $\tilde{y}_{1}(k)$ and quantified output signal $\bar{y}_{1}(k)$.

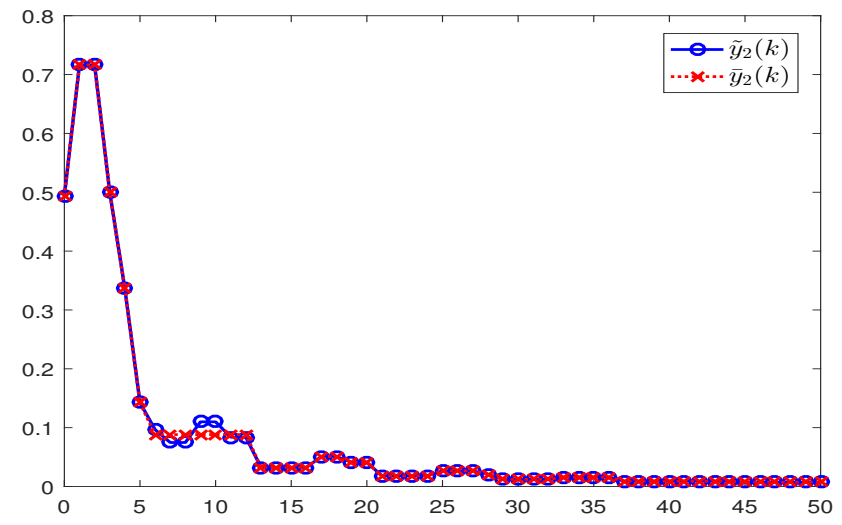

Figure 9. The dynamic event-triggered output signal $\tilde{y}_{2}(k)$ and quantified output signal $\bar{y}_{2}(k)$. 


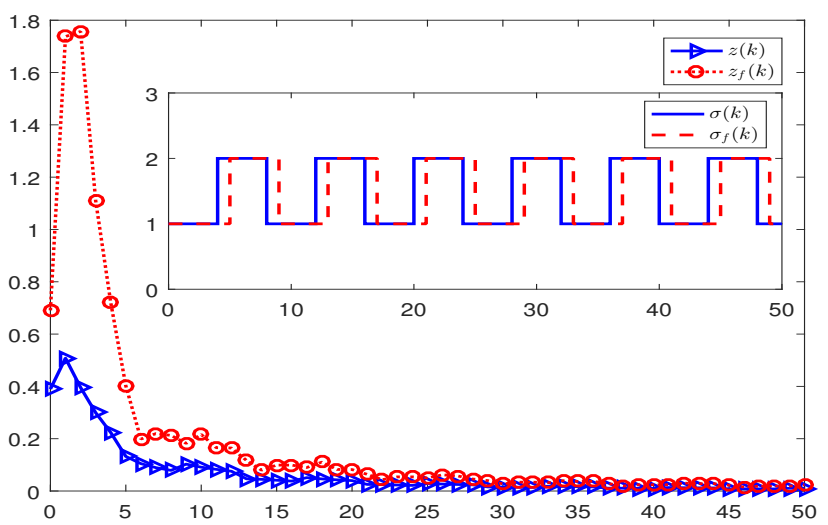

Figure 10. The simulations of $z(k)$ and $z_{f}(k)$ with asynchronous switching signal.

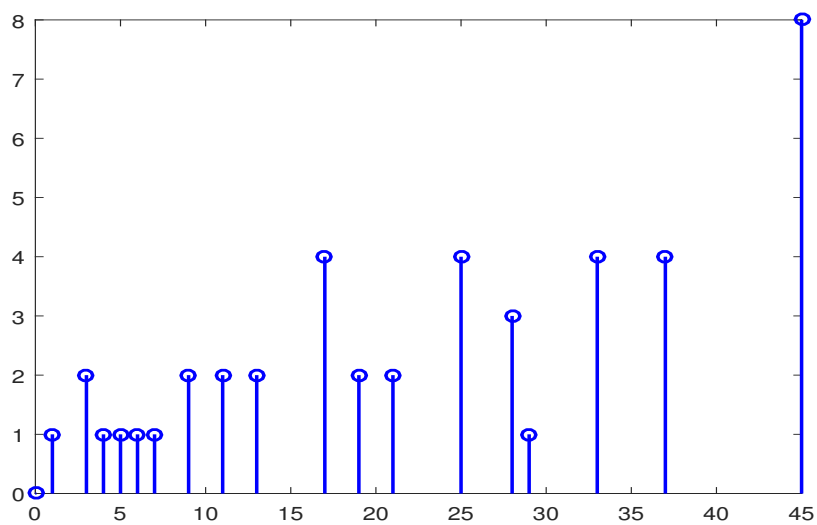

Figure 11. The dynamic event-triggering release instants and release intervals.

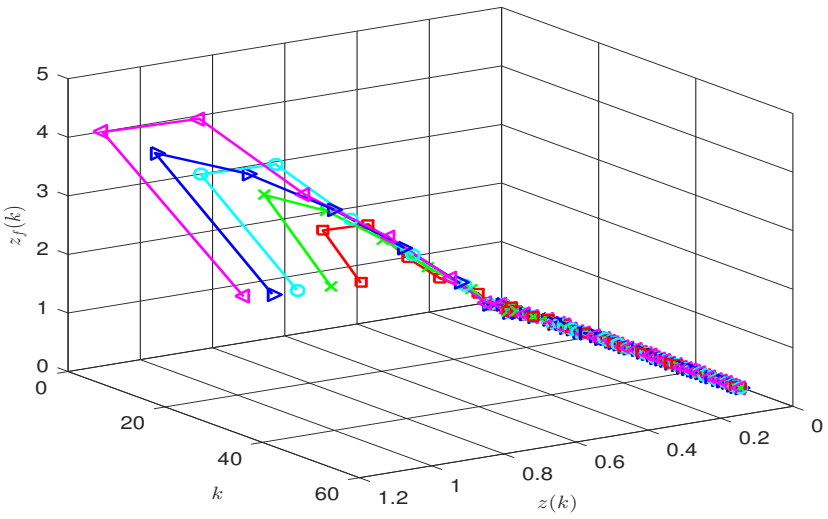

Figure 12. The simulations of $z(k)$ and $z_{f}(k)$ under different initial conditions.

\section{Conclusions}

In this paper, we investigated an event-triggered asynchronous filter of nonlinear switched positive systems with output quantization. Based on static and dynamic eventtriggering mechanisms, an asynchronous filter was proposed using the matrix decomposition technique. The positivity and $L_{1}$-gain stability of the underlying systems were guaranteed by using a linear copositive Lyapunov function and linear programming approach. Then, the issue of output quantization is solved under a quantizer.

Author Contributions: Conceptualization, J.Z. and P.L.; methodology, P.L.; software, P.L.; validation, S.Z. and P.L.; formal analysis, J.Z.; investigation, S.Z., P.L. and J.Z.; resources, S.Z. and P.L.; data curation, P.L.; writing - original draft preparation, S.Z. and P.L.; writing—review and editing, J.Z.; visualization, S.Z. and P.L.; supervision, J.Z.; project administration, P.L. and J.Z. All authors have read and agreed to the published version of the manuscript. 
Funding: This work was supported by the Fundamental Research Funds for the Provincial Universities of Zhejiang (Nos. GK219909299001-002, GK219909299001-403, and GK209907299001-007), the National Natural Science Foundation of China (No. 62003119), and the Natural Science Foundation of Zhejiang Province, China (No. LQ21F030014).

Institutional Review Board Statement: Not applicable.

Informed Consent Statement: Not applicable.

Data Availability Statement: Not applicable.

Conflicts of Interest: The authors declare no conflict of interest. The funders had no role in the design of the study; in the collection, analyses, or interpretation of data; in the writing of the manuscript; or in the decision to publish the results.

\section{References}

1. Farina, L.; Rinaldi, S. Positive Linear Systems: Theory and Applications; John Wiley \& Sons: Hoboken, NJ, USA, 2000.

2. Kaczorek, T. Positive 1D and 2D Systems; Springer: London, UK, 2001.

3. Hespanha J.P.; Morse A.S. Stability of switched systems with average dwell-time. In Proceedings of the 38th IEEE Conference on Decision and Control, Phoenix, AZ, USA, 7-10 December 1999; Volume 3, pp. 2655-2660.

4. Dinh, T.N.; Marouani, G.; Raïssi, T.; Wang, Z.; Messaoud, H. Optimal interval observers for discrete-time linear switched systems. Int. J. Control 2020, 93, 2613-2621. [CrossRef]

5. Jadbabaie, A.; Lin, J.; Morse, A. Coordination of groups of mobile autonomous agents using nearest neighbor rules. IEEE Trans. Autom. Control 2003, 48, 988-1001. [CrossRef]

6. Liberzon, D. Switching in Systems and Control; Springer: Berlin/Heidelberg, Germany, 2003.

7. Silva-Navarro, G.; Alvarez-Gallegos, J. On the property sign-stability of equilibria in quasimonotone positive nonlinear systems. In Proceedings of 1994 33rd IEEE Conference on Decision and Control, Lake Buena Vista, FL, USA, 14-16 December 1994; Volume 4, pp. 4043-4048.

8. Ocampo-Martinez, C.; Puig, V.; Cembrano, G.; Quevedo, J. Application of predictive control strategies to the management of complex networks in the urban water cycle. IEEE Control. Syst. Mag. 2013, 33, 15-41.

9. Fornasini, E.; Valcher, M.E. Stability and stabilizability criteria for discrete-time positive switched systems. IEEE Trans. Autom. Control 2011, 57, 1208-1221. [CrossRef]

10. Pastravanu, O.C.; Matcovschi, M.H. Max-type copositive Lyapunov functions for switching positive linear systems. Automatica 2014, 50, 3323-3327. [CrossRef]

11. Xiang, W.; Lam, J.; Shen, J. Stability analysis and $L_{1}$-gain characterization for switched positive systems under dwell-time constraint. Automatica 2017, 85, 1-8. [CrossRef]

12. Zhang, J.; Han, Z.; Zhu, F. L1-gain analysis and control synthesis of positive switched systems. Int. J. Syst. Sci. 2015, 46, $2111-2121$. [CrossRef]

13. Fainshil, L.; Margaliot, M.; Chigansky, P. On the stability of positive linear switched systems under arbitrary switching laws. IEEE Trans. Autom. Control 2009, 54, 897-899. [CrossRef]

14. Blanchini, F.; Colaneri, P.; Valcher, M.E. Co-positive Lyapunov functions for the stabilization of positive switched systems. IEEE Trans. Autom. Control 2012, 57, 3038-3050. [CrossRef]

15. Xiang, M.; Xiang, Z.; Karimi, H.R. Stabilization of positive switched systems with time-varying delays under asynchronous switching. Int. J. Control. Autom. Syst. 2014, 512, 939-947. [CrossRef]

16. Wang, D.; Wang, Z.; Li, G.; Wang, W. Distributed filtering for switched nonlinear positive systems with missing measurements over sensor networks. IEEE Sens. J. 2016, 16, 4940-4948. [CrossRef]

17. Zhang, J.; Zhao, X.; Cai, X. Absolute exponential $L_{1}$-gain analysis and synthesis of switched nonlinear positive systems with time-varying delay. Appl. Math. Comput. 2016, 284, 24-36.

18. Zhang, J.; Raïssi, T. Saturation control of switched nonlinear systems. Nonlinear Anal. Hybrid Syst. 2019, 32, 320-336. [CrossRef]

19. Liu, X.; Yuan, S. Reduced-order fault detection filter design for switched nonlinear systems with time delay. Nonlinear Dyn. 2012, 67, 601-617. [CrossRef]

20. Zheng, Q.; Zhang, H. $H_{\infty}$ filtering for a class of nonlinear switched systems with stable and unstable subsystems. Signal Process. 2017, 141, 240-248. [CrossRef]

21. Aleksandrov, A.; Aleksandrova, E.; Zhabko, A. Stability analysis of some classes of nonlinear switched systems with time delay. Int. J. Syst. Sci. 2017, 48, 2111-2119. [CrossRef]

22. Baleghi, N.A.; Shafiei, M.H. Stability analysis and stabilization of a class of discrete-time nonlinear switched systems with time-delay and affine parametric uncertainty. J. Vib. Control 2019, 25, 1326-1340. [CrossRef]

23. Huo, X.; Ma, L.; Zhao, X.; Zong, G. Observer-based fuzzy adaptive stabilization of uncertain switched stochastic nonlinear systems with input quantization. J. Frankl. Inst. 2019, 356 1789-1809. [CrossRef]

24. Yang, J.; Chen, Y.; Zheng, Z.; Qian, W. Robust adaptive state estimation for uncertain nonlinear switched systems with unknown inputs. Trans. Inst. Meas. Control. 2018, 40, 1082-1091. [CrossRef] 
25. Niu, B.; Zhao, P.; Liu, J.D.; Ma, H.J.; Liu, Y.J. Global adaptive control of switched uncertain nonlinear systems: An improved MDADT method. Automatica 2020, 115, 108872. [CrossRef]

26. Xiang, W.; Xiao, J. $H_{\infty}$ filtering for switched nonlinear systems under asynchronous switching. Int. J. Syst. Sci. 2011, 42, 751-765. [CrossRef]

27. Zheng, Q.; Xu, S.; Zhang, Z. Asynchronous nonfragile $H_{\infty}$ filtering for discrete-time nonlinear switched systems with quantization. Nonlinear Anal. Hybrid Syst. 2020, 37, 100911. [CrossRef]

28. Ren, Y.; Er M.J.; Sun, G. Asynchronous $\ell_{1}$ positive filter design for switched positive systems with overlapped detection delay IET Control Theory Appl. 2017, 11, 319-328. [CrossRef]

29. Wang, B.; Zhang, H.; Wang, G.; Dang, C. Asynchronous $H_{\infty}$ filtering for linear switched systems with average dwell time. Int. J. Syst. Sci. 2016, 47, 2783-2791. [CrossRef]

30. Li, Y.; Du, W.; Xu, X.; Zhang, H.; Xia, J. A novel approach to $L_{1}$ filter design for asynchronously switched positive linear systems with dwell time. Int. J. Robust Nonlinear Control 2019, 29, 5957-5978. [CrossRef]

31. Brockett, R.W.; Liberzon, D. Quantized feedback stabilization of linear systems. IEEE Trans. Autom. Control 2000, 45, 1279-1289. [CrossRef]

32. Wakaiki, M.; Yamamoto, Y. Stabilization of switched linear systems with quantized output and switching delays. IEEE Trans Autom. Control 2016, 62, 2958-2964. [CrossRef]

33. Cheng, J.; Park, J. H.; Cao, J.; Zhang, D. Quantized $H_{\infty}$ filtering for switched linear parameter-varying systems with sojourn probabilities and unreliable communication channels. Inf. Sci. 2018, 466, 289-302. [CrossRef]

34. Yang, L.; Guan, C.; Fei, Z. Finite-time asynchronous filtering for switched linear systems with an event-triggered mechanism. J. Frankl. Inst. 2019, 356, 5503-5520. [CrossRef]

35. Zong, G.; Ren, H.; Karimi, H.R. Event-triggered communication and annular finite-time $H_{\infty}$ filtering for networked switched systems. IEEE Trans. Cybern. 2020, 51, 309-317. [CrossRef]

36. Liu, X.; Su, X.; Shi, P.; Nguang, S.K.; Shen, C. Fault detection filtering for nonlinear switched systems via event-triggered communication approach. Automatica 2019, 101, 365-376. [CrossRef]

37. Wang, Y.L.; Shi, P.; Lim, C.C.; Liu, Y. Event-triggered fault detection filter design for a continuous-time networked control system. IEEE Trans. Cybern. 2016, 46, 3414-3426. [CrossRef] [PubMed]

38. Zhang, J.; Zheng, G.; Feng, Y.; Chen, Y. Event-triggered state-feedback and dynamic output-feedback control of positive Markovian jump systems with intermittent faults. IEEE Trans. Autom. Control 2022. [CrossRef]

39. Xiao, S.; Zhang, Y.; Xu, Q.; Zhang, B. Event-triggered network-based $L_{1}$-gain filtering for positive linear systems. Int. J. Syst. Sci. 2017, 48, 1281-1290. [CrossRef]

40. Zhang, J.; Raïssi, T.; Shao, Y.; Cai, X. Event-triggered filter design of positive systems with state saturation. IEEE Syst. J. 2020, 15, 4281-4285. [CrossRef]

41. Shao, Y.; Jiao, C.; Zhang, S.; Zhang, J. Event-triggered filter of switched positive systems with state saturation. In Proceedings of the 2020 7th International Conference on Information, Cybernetics, and Computational Social Systems, Guangzhou, China, 13-15 November 2020; pp. 1-6.

42. Girard, A. Dynamic triggering mechanisms for event-triggered control. IEEE Trans. Autom. Control 2014, 60, 1992-1997. [CrossRef]

43. Gu, Z.; Tian, E.; Liu, J. Adaptive event-triggered control of a class of nonlinear networked systems. J. Frankl. Inst. 2017, 354, 3854-3871. [CrossRef]

44. Li, Q.; Shen, B.; Wang, Z.; Sheng, W. Recursive distributed filtering over sensor networks on Gilbert-Elliott channels: A dynamic event-triggered approach. Automatica 2020, 113, 108681. [CrossRef]

45. Liu, D.; Yang, G.H. Dynamic event-triggered control for linear time-invariant systems with-gain performance. Int. J. Robust Nonlinear Control 2019, 29, 507-518. [CrossRef]

46. Luo, S.; Deng, F.; Chen, W.H. Dynamic event-triggered control for linear stochastic systems with sporadic measurements and communication delays. Automatica 2019, 107, 86-94. [CrossRef]

47. Lu, R.; Wu, F.; Xue, A. Networked control with reset quantized state based on Bernoulli processing. IEEE Trans. Ind. Electron. 2013, 61, 4838-4846. [CrossRef]

48. Dong, S.; Su, H.; Shi, P.; Lu, R.; Wu, Z.G. Filtering for discrete-time switched fuzzy systems with quantization. IEEE Trans. Fuzzy Syst. 2016, 25, 1616-1628. [CrossRef]

49. Wang, Y.; Han, Q. Network-based modelling and dynamic output feedback control for unmanned marine vehicles in network environments. Automatica 2018, 91, 43-53. [CrossRef] 